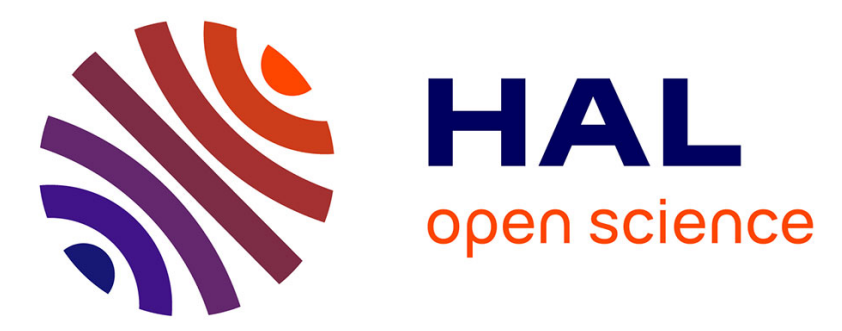

\title{
More room for microphase separation: An extended study on binary liquids confined in SBA-15 cylindrical pores
}

Ramona Mhanna, Abdel Razzak Abdel Hamid, Sujeet Dutta, Ronan Lefort, Laurence Noirez, Bernhard Frick, Denis Morineau

\section{To cite this version:}

Ramona Mhanna, Abdel Razzak Abdel Hamid, Sujeet Dutta, Ronan Lefort, Laurence Noirez, et al. More room for microphase separation: An extended study on binary liquids confined in SBA15 cylindrical pores. Journal of Chemical Physics, 2017, 146 (2), pp.024501. 10.1063/1.4972126 . hal-01405997

\section{HAL Id: hal-01405997 \\ https://hal.science/hal-01405997}

Submitted on 5 Dec 2016

HAL is a multi-disciplinary open access archive for the deposit and dissemination of scientific research documents, whether they are published or not. The documents may come from teaching and research institutions in France or abroad, or from public or private research centers.
L'archive ouverte pluridisciplinaire HAL, est destinée au dépôt et à la diffusion de documents scientifiques de niveau recherche, publiés ou non, émanant des établissements d'enseignement et de recherche français ou étrangers, des laboratoires publics ou privés. 


\section{More room for microphase separation: An extended}

\section{study on binary liquids confined in SBA-15}

\section{cylindrical pores}

Ramona Mhanna, ${ }^{1,3}$ Abdel Razzak Abdel Hamid, ${ }^{1}$ Sujeet Dutta, ${ }^{1}$ Ronan Lefort, ${ }^{1}$ Laurence Noirez, ${ }^{2}$

Bernhard Frick ${ }^{3}$, Denis Morineau ${ }^{1, a)}$

${ }^{1}$ Institute of Physics of Rennes, CNRS-University of Rennes 1, UMR 6251, F-35042 Rennes,

France.

${ }^{2}$ Laboratoire Léon Brillouin (CEA-CNRS), UMR12, Université Paris-Saclay, CE-Saclay F91191 Gif-sur-Yvette, France

${ }^{3}$ Institut Laue-Langevin, 71 avenue des Martyrs, F-38000 Grenoble, France

a) Corresponding Author: denis.morineau@univ-rennes1.fr 


\section{ABSTRACT}

The confinement of liquid mixtures in porous channels provides new insight into fluid ordering at the nanoscale. In this study, we address a phenomenon of microphase separation, which appears as a novel fascinating confinement effect for fully miscible binary liquids. We investigate the structure of tert-butanol-toluene mixtures confined in the straight and mono-dispersed cylindrical nanochannels of SBA-15 mesoporous silicates $(D=8.3 \mathrm{~nm})$. Small angle neutron scattering experiments on samples with carefully designed isotopic compositions are performed to systematically vary the scattering length density of the different compounds and assess the radial concentration profile of the confined phases. The resulting modulation of the Bragg reflections of SBA-15 is compared with the predictions from different core-shell models, highlighting a molecular-scale phase-separated tubular structure with the tert-butanol forming a layer at the pore surface, surrounding a toluene-rich core. The present structural study suggests that the microphase separation phenomenon in confinement, which so far had only been reported for a smaller pore size $(D=3.65 \mathrm{~nm})$ and a unique mixture composition must be considered as a general phenomenon. It also highlights the strength of neutron scattering method with isotopic substitution, which is a unique experimental approach to reveal this phenomenon. 


\section{INTRODUCTION}

In the past decades, the modification of the properties of matter at the nanoscale has been addressed through the impregnation of molecular fluids into the nanochannels of mesoporous structures. These studies have shown that the structural and dynamical properties under spatial confinement could not be accounted in terms of the bulk analogs due to the strong interfacial and finite size effects that dominate on scales comparable to a few molecular diameters. ${ }^{1-9}$ In particular, the formation of original structures of simple liquids in confined geometry has been intensively investigated..$^{6,7,9-13}$ The influence of the confinement has been extended to the dynamics of binary liquids, providing indirect evidence for a microphase separation of fully miscible mixtures of alcohol aqueous mixtures confined in MCM-41 mesostructured porous silicates. ${ }^{14-18}$

In a recent study, we conducted a thorough structural analysis to characterize the nature of microphase-separated systems. ${ }^{19,20}$ We determined the concentration profile across the nanochannels by implementing an original application of neutron diffraction properties with isotopic substitution and derived a quantitative model to characterize the binary liquid structure. We concluded on the existence of a novel surface-induced core-shell structure for fully miscible tert-butanol (TBA)-toluene (TOL) binary mixtures confined in MCM-41 silicates with a pore size $D=3.65 \mathrm{~nm}$. In this system, tert-butanol segregates as a pore surface layer of about one atomic size surrounding a toluene-rich core of two to three atomic layers. The preferential segregation of the alcohol at the surface illustrates the amphiphilic character of TBA, supported by the observation of supramolecular clusters in the bulk TBA-TOL mixtures, ${ }^{21}$ and the higher affinity of alcohol to silica, which was demonstrated by binary gas adsorption ${ }^{22}$ and by the formation of interfacial H-bonded alcohol clusters in alcohol-alkane mixtures in contact with hydrophilic surfaces. ${ }^{23-27}$ 
The aim of the present study is to question the generalization of this microphase-separation concept. Can we observe such phenomenon for a different pore size and up to which length scale does it extend? Is the phenomenon limited to a specific mixture (i.e. with a volume fraction around 50/50) or can it be observed for a wider range of compositions? To tackle such questions we use SBA-15 silicate hosts which are quite interesting for addressing the question of the pore size effects, because they are rather similar to MCM-41 mesoporous silicates with a 2-D long range triangular order of cylindrical channels but with a pore diameter that is twice as large $(D=8.3$ $\mathrm{nm})$. Moreover, changing the mixture composition proved to be an efficient and straightforward way to check the domain of stability of the microphase separated structure and to assess the corresponding core and shell proportions.

\section{EXPERIMENTAL DETAILS}

Hydrogenated solvents TBA and TOL $(>99 \%)$ and fully deuterated solvents TBA $\left(\mathrm{C}_{4} \mathrm{D}_{10} \mathrm{O}\right.$, 99.8\%) and TOL $\left(\mathrm{C}_{7} \mathrm{D}_{8}, 99.5 \%\right)$ were purchased from Sigma-Aldrich and from Eurisotop respectively, and used directly without further purification. The mesoporous silicates SBA-15 were prepared in our laboratory according to a procedure similar to that described elsewhere ${ }^{28}$ with minor modifications in the thermal treatments to optimize the final structure of the product. ${ }^{29}$ Nonionic triblock copolymer (Pluronic $\left.\mathrm{P}_{123}\right)$ : $(\mathrm{EO})_{20}(\mathrm{PO})_{70}(\mathrm{EO})_{20}$ was used as template to get a mesostructured triangular array of aligned channels with pore diameter $D=8.3 \mathrm{~nm}$, as confirmed by nitrogen adsorption, transmission electron microscopy and neutron diffraction. The calcined matrix was dried at $120^{\circ} \mathrm{C}$ under primary vacuum for 12 hours prior to the neutron experiments. The neutron diffraction experiments were performed on the PAXY small angle neutron scattering diffractometer at the Laboratoire Léon Brillouin (CEA-CNRS Saclay). The chosen sample detector 
distance was $1.6 \mathrm{~m}$ and the neutron wavelength $0.6 \mathrm{~nm}$, allowing to measure the diffracted intensity in a momentum transfer i.e. $q$-range from 0.3 to $3 \mathrm{~nm}^{-1}$. The measurements were conducted at room temperature with a thermal bath regulator set to $22^{\circ} \mathrm{C}$. The empty SBA- 15 was packed in $1 \mathrm{~mm}$ thick quartz Hellma cells, and then filled by liquid imbibition with the appropriate amount of TBATOL mixtures injected in the sealed cells from a syringe to allow complete loading of the porous volume $V_{P}=1.02 \mathrm{~cm}^{3} \mathrm{~g}^{-1}$, measured by nitrogen adsorption. The neutron scattering measurements were started about one hour after the liquid injection, and the spectra were acquired periodically (typically one spectrum per sample every 1.5 hour) for a total period of about 15 hours. This allowed monitoring the porous matrix imbibition process in real-time and confirmed the complete filling of the porosity from the time evolution of the spectra. The sample mass was verified after the neutron scattering measurements to confirm the absence of any evaporation from the sealed cell. The intensities were corrected from empty cell contribution by subtraction of the filled and empty sample spectra, divided by their own measured forward transmission. The correction of the detector efficiency and normalization were performed with respect to the measured flat intensity of hydrogenated water filled in the same cell.

\section{RESULTS}

In this manuscript, we develop a procedure which is adapted from the previous work on MCM41 materials, and based on the study of two sets of experiments. ${ }^{19}$ The first set of results comprises different types of contrast matching liquids (i.e. unary and binary mixtures), the second different mixture compositions, which fulfill selective contrast matching conditions.

\section{A. Contrast matching liquids}


The diffraction pattern of the empty SBA-15 host exhibits Bragg reflections at relatively small angle vector, due to the regular large lattice spacing of the pores which can be indexed by the Miller indices ( $h k$ ), using as base a triangular 2-D lattice (cf. Fig. 1). In the case of a homogeneous pore filling with a constant density profile across the pore, the intensity of the Bragg reflections $I_{h k}^{\text {filled }}$ of the filled samples are related to the intensity $I_{h k}^{\text {empty }}$ of the empty SBA-15 by a scaling factor defined as:

$$
\frac{I_{h k}^{\text {filled }}}{I_{h k}^{\text {empty }}}=\frac{\left[(n \bar{b})_{\mathrm{SiO}_{2}}-(n \bar{b})_{\text {liquid }}\right]^{2}}{\left[(n \bar{b})_{\mathrm{SiO}_{2}}-0\right]^{2}}
$$

where $n$ is the number density of the molecules within the pore, $\bar{b}$ is the sum over the coherent scattering lengths of all atoms within the molecule and $(n \bar{b})$ is the molecular scattering length density. ${ }^{19,20,30}$ Therefore a complete extinction of the Bragg peaks intensity is expected when $(n \bar{b})_{\text {SiO }_{2}}=(n \bar{b})_{\text {liquid }}$. This situation is realized when the matrix is filled completely with a contrast matching liquid, i.e. the pore filling liquid has the same average scattering length density as silica, which can be realized by varying the $\mathrm{D} / \mathrm{H}$ isotopic composition of the liquid. ${ }^{19} \mathrm{We}$ used $54 \% \mathrm{D} / \mathrm{H}$ volume fraction for TOL and $55 \% \mathrm{D} / \mathrm{H}$ for TBA to obtain compositions that fulfill an almost perfect contrast matching condition with $(n \bar{b})_{\text {SiO }_{2}}=(n \bar{b})_{\text {liquid }}=34.6 \times 10^{9} \mathrm{~cm}^{-2}$ assuming that the matrix consists of amorphous silica $\left(2.2 \mathrm{~g} . \mathrm{cm}^{-3}\right)$. 


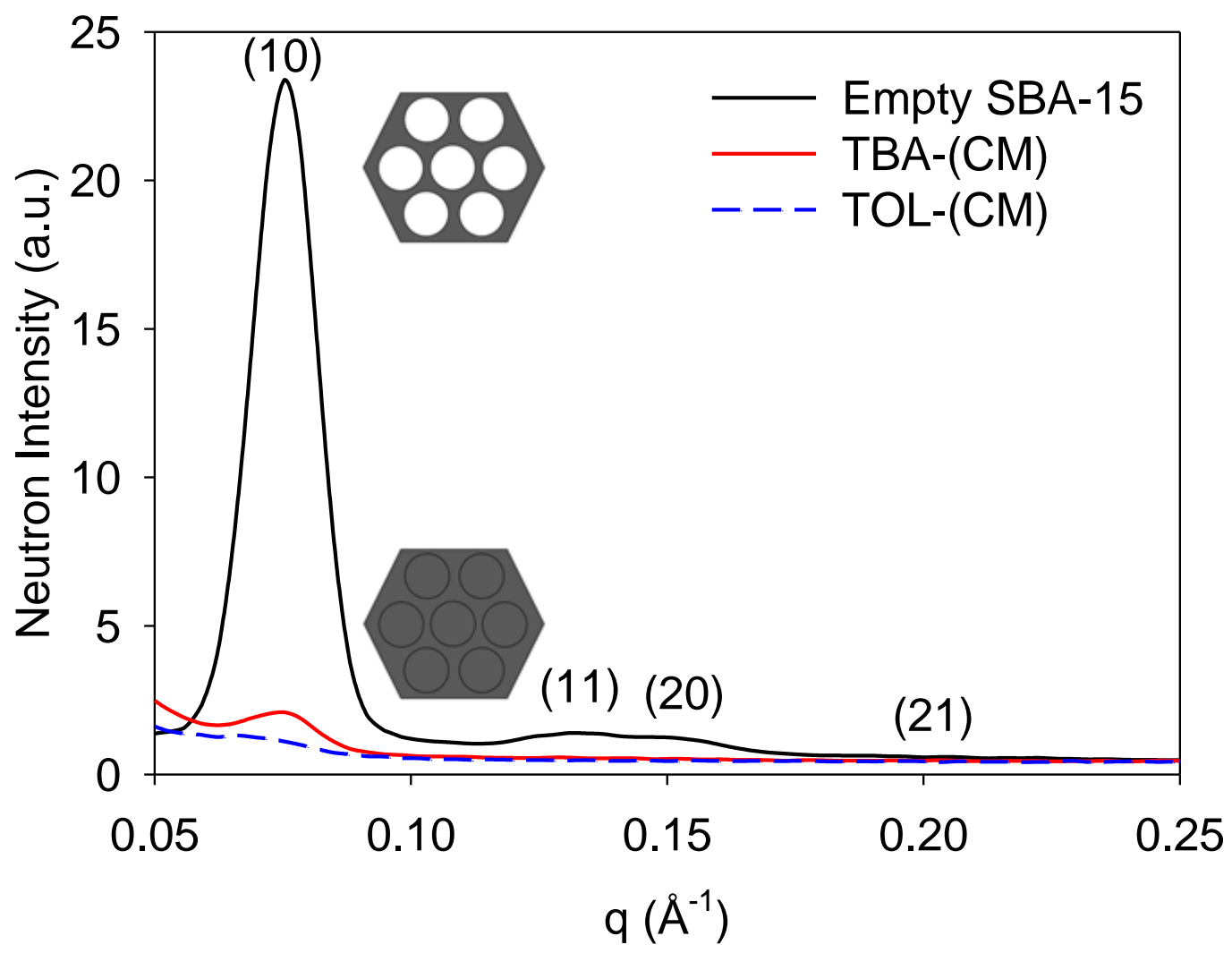

FIG. 1. Neutron diffraction patterns of empty SBA-15 (solid black line) and SBA-15 filled with contrast matching isotopic mixtures comprising only tert-butanol (solid red line) and only toluene (dashed blue line) after $15 \mathrm{~h}$ equilibration. The Bragg reflections of SBA-15 are labelled with Miller indices $(h k)$. Inset: sketches corresponding to the empty SBA-15 (top inset), SBA-15 filled with contrast matching liquid (bottom inset). 


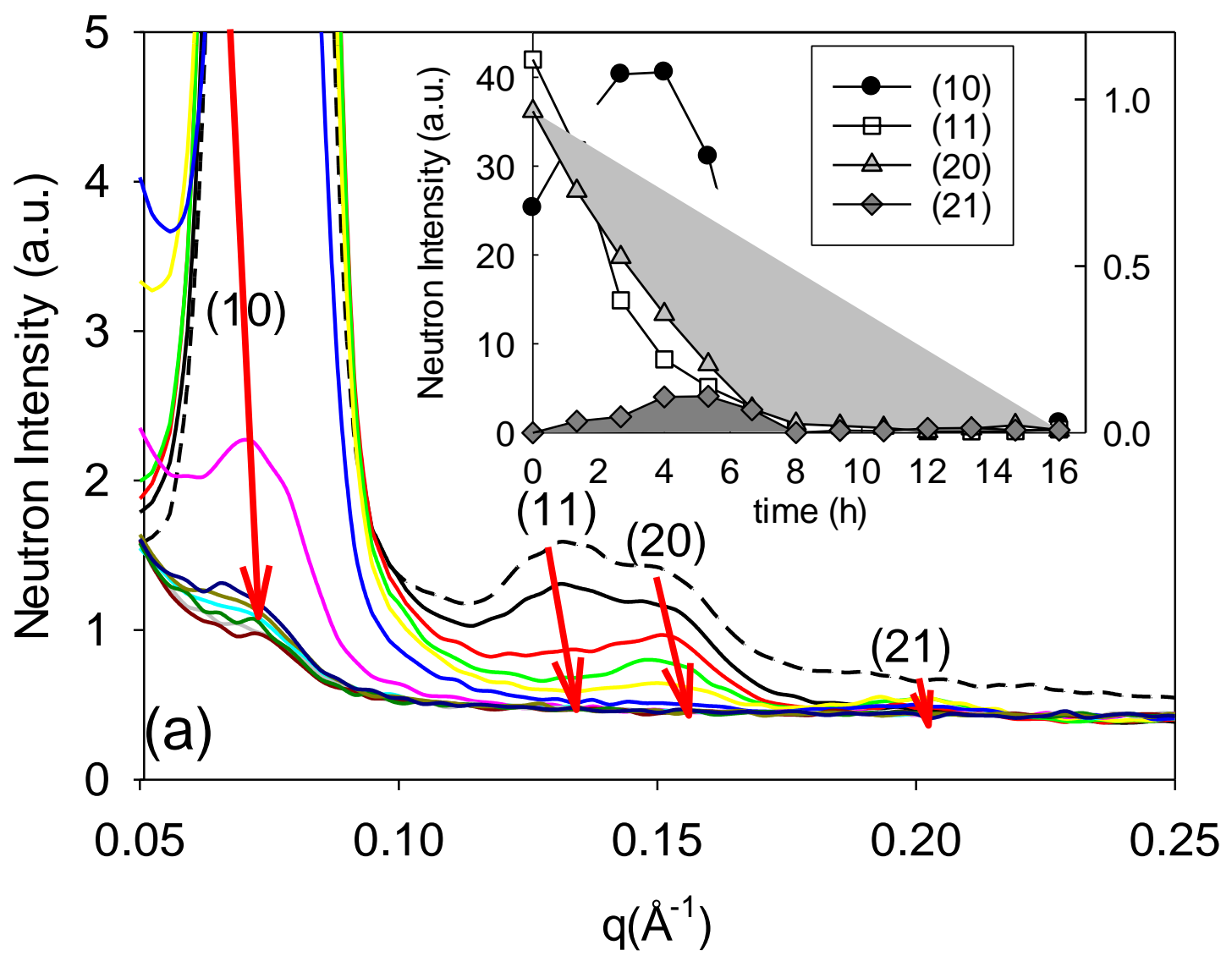




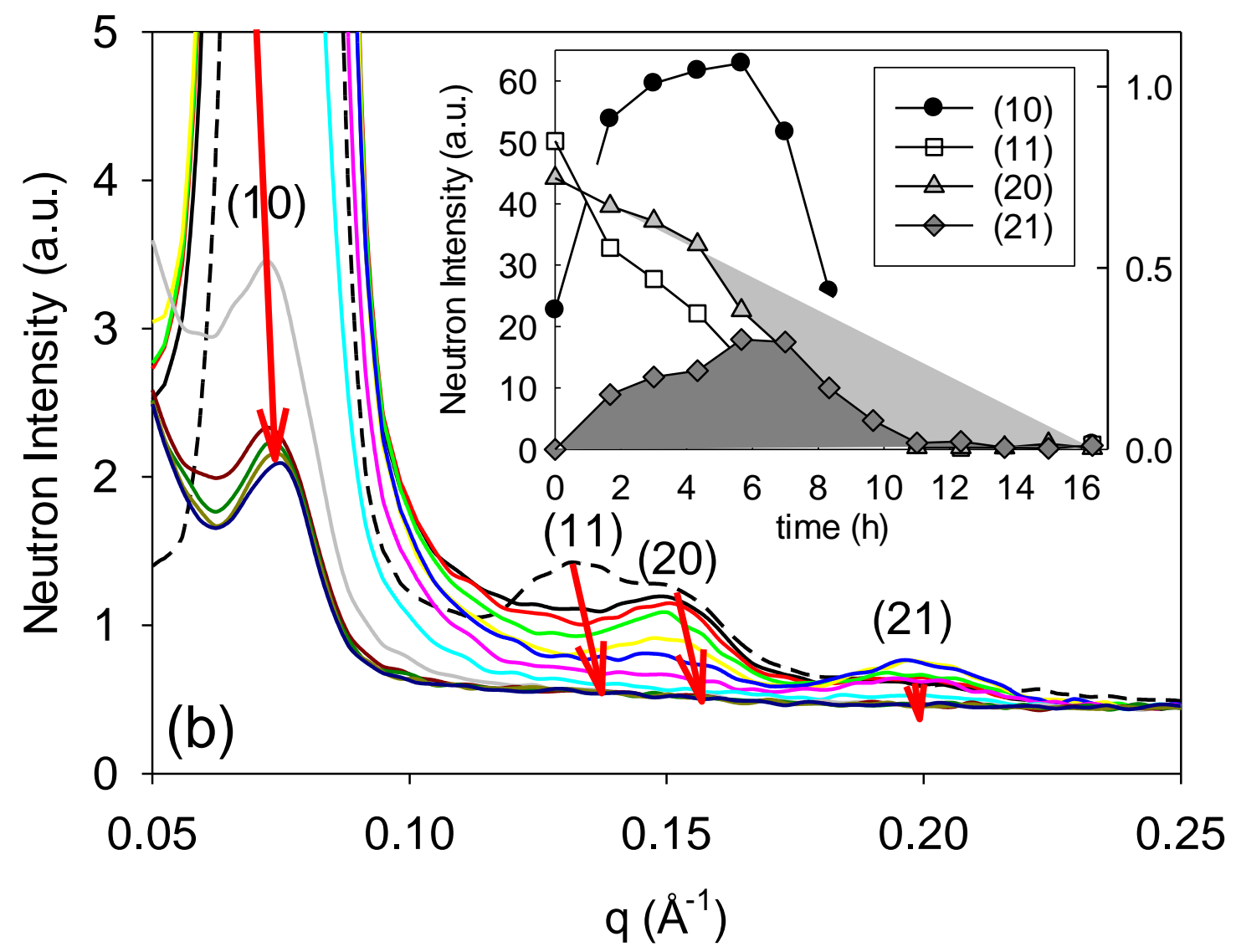

FIG. 2. Time evolution of the neutron diffraction patterns of empty SBA-15 (dashed line), and SBA-15 filled with (a) contrast matching isotopic mixtures comprising only toluene and (b) tertbutanol. The red arrows point from the Bragg reflections of the first spectrum towards the last one. Refer to the inset for the time correspondence of each spectrum. Inset: time evolution of the Bragg peaks maximum intensity (refer to the left y-axis for the (10) peak and to the right y-axis for the others). 
The scattering intensity of the pure contrast matching TOL and TBA in SBA-15 is shown in Fig. 2 (a) and Fig. 2 (b) as a function of time after injection of the liquid within the sealed cell containing the dry porous matrix. The relative intensity of the Bragg reflections ((10), (11), (20) and (21)) varies rapidly after injection and finally reaches constant values. Their positions remain constant, which demonstrates that the crystalline structure of the pores is unchanged by liquid confinement. It is not excluded, and even likely, that the mesoporous material is deformed elastically by the action of capillary forces, but according to the literature the strain of the order of $10^{-4}$ to $10^{-3}$ would be much too small to be observed in our SANS experiment using a wavelength rotating selector with a resolution of about $10 \%{ }^{31,32}$ Three important conclusions can be derived from these realtime experiments: (1) using this imbibition method, it appears that a delay of about 10 hours is required to reach an equilibrium state that corresponds to the total imbibition of the porous matrix. This result is consistent with previous studies. ${ }^{19}$ (2) The variation of the Bragg peaks, viz. (10) and (21), is non-monotonous, and exhibits maximum values for intermediate times. A possible explanation is the faster adsorption of molecules within the silica microporosity that results in a larger density contrast between the empty porous volume and matrix walls, and so a higher scattered intensity than for the corresponding empty matrix. It is worth pointing out that the dynamics of pore filling, related to a slow and non-monotonic equilibration of the diffraction pattern, possibly depends on many experimental parameters, which are specific to the material (pore size, grain size c.a. $1 \mu \mathrm{m}$, surface chemistry...), but also to the experimental conditions (powder packing, sample cell size...). It highlights the significance of further studies on the kinetics of pore filling to assess the microscopic mechanisms of liquid imbibition and/or vapor adsorption of binary systems, expecting composition change during capillary condensation, ${ }^{22}$ through in situ adsorption experiments. (3) An almost total extinction of the structure factor is 
eventually observed, which unambiguously indicates the homogeneous and complete filling by capillary condensation of the liquids in the pore under contrast matching conditions. 


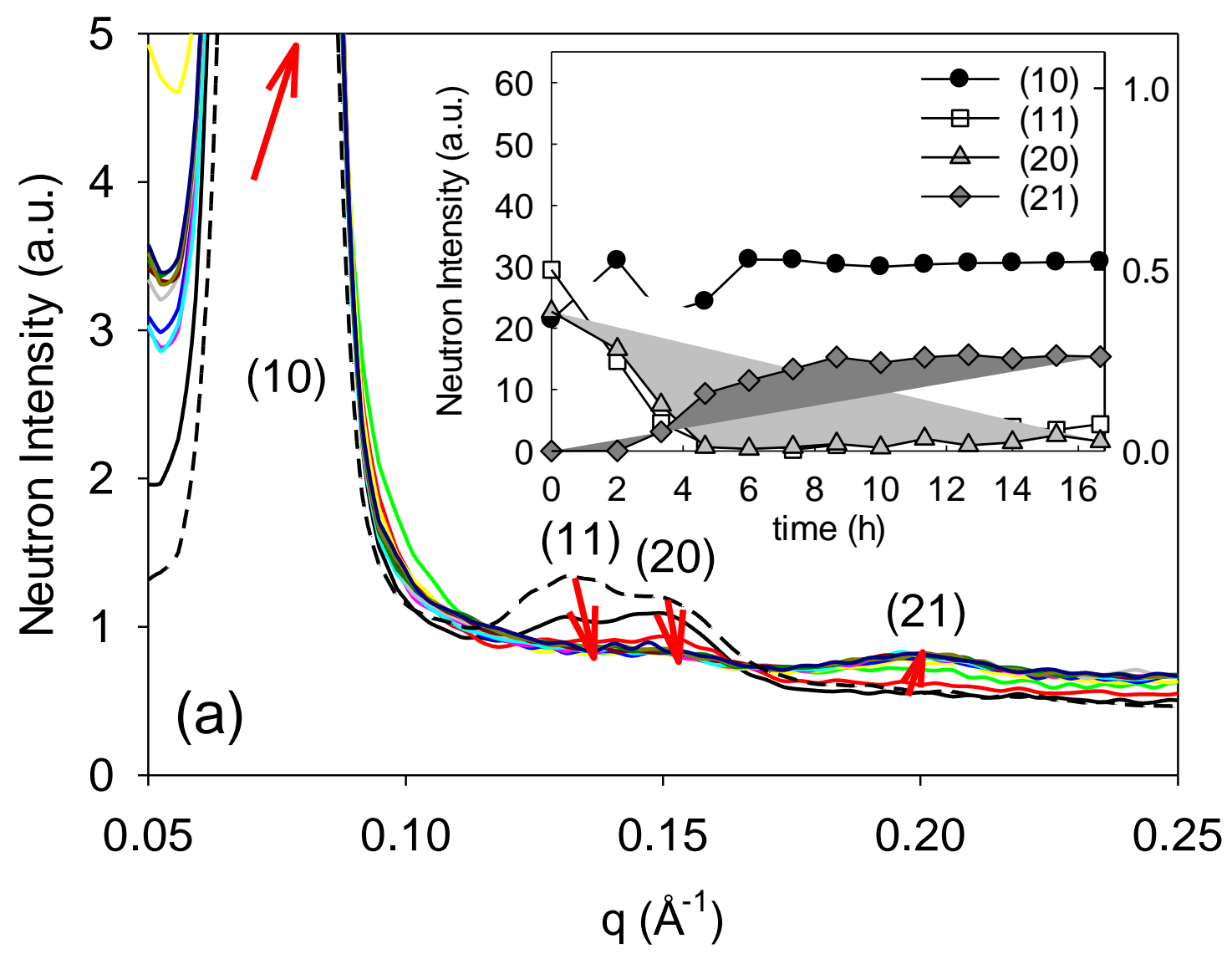




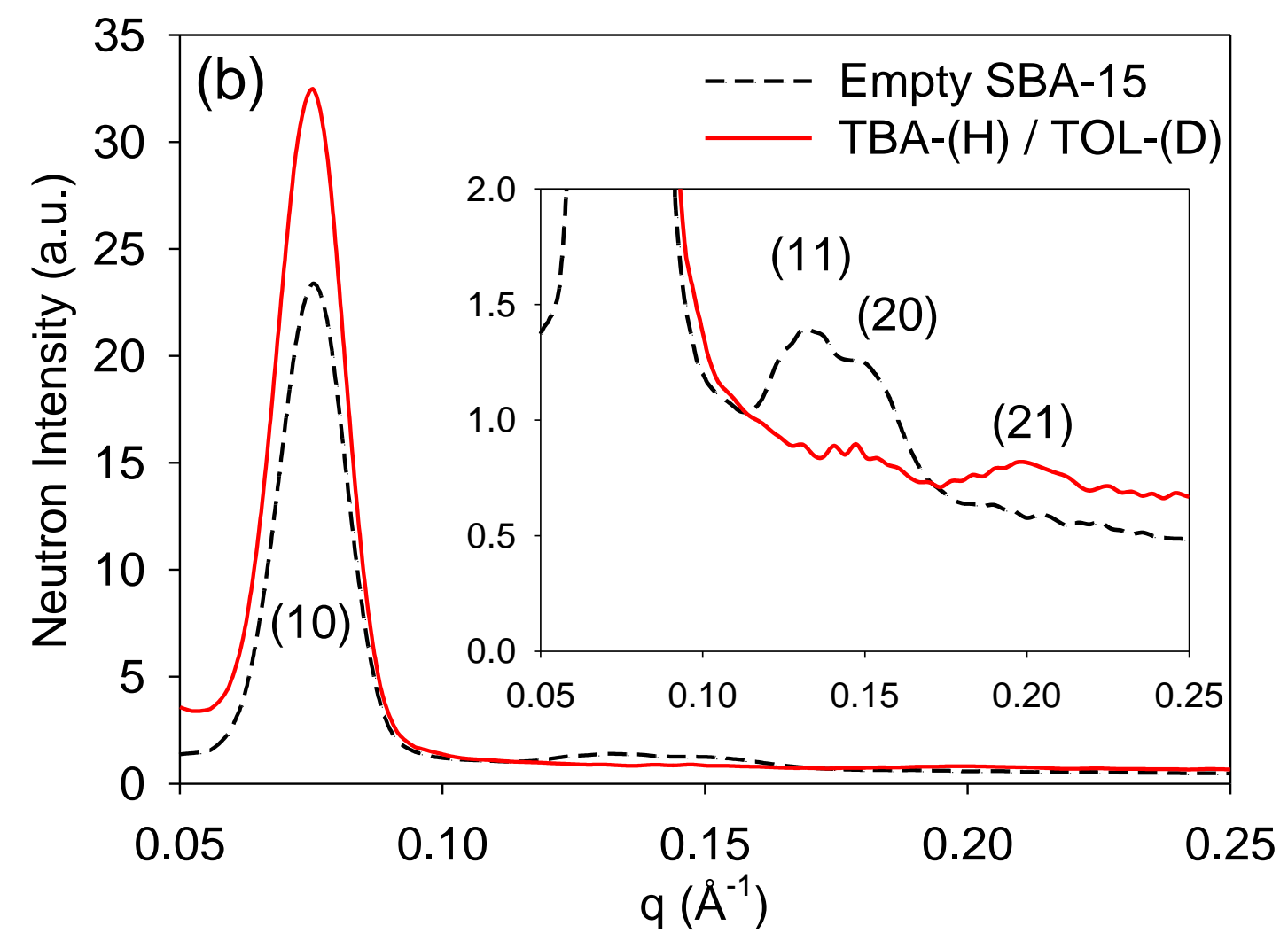

FIG. 3. (a) Time evolution of the neutron diffraction patterns of empty SBA-15 (dashed line), and SBA-15 filled with a contrast matching isotopic mixtures comprising hydrogenated tert-butanol with deuterated toluene (volume fraction 37/63\%). The red arrows point from the Bragg reflections of the first spectrum towards the last one. Refer to the inset for the time correspondence of each spectrum. Inset: time evolution of the Bragg peaks maximum intensity (refer to the left y-axis for the (10) peak and right y-axis for the others). (b) Neutron diffraction patterns of empty SBA-15 (black dashed line) and filled SBA-15 (red line) after $15 \mathrm{~h}$ equilibration. Inset: Detail of the higher Bragg orders. 
A first direct evidence for the microphase separation of the TBA-TOL mixture in the SBA-15 nanochannels is obtained with a binary liquid comprising hydrogenated TBA-(H) with deuterated TOL-(D) with a volume composition 37/63, so that it fulfills the contrast matching condition. Indeed, Fig. 3 (b) shows that the extinction of the structure factor of the pore lattice is not achieved under these circumstances. From the time evolution of the scattered intensity (inset of Fig. 3 (a)), the imbibition process is characterized by the decrease and the eventual total extinction of two Bragg peaks indexed (11) and (20). But at the same time the intensity of the (10) peak increases, reaching a final value which is as high as 1.5 times that of the empty SBA-15. This uncommon behavior is also observed for the reflection (21), which was negligible for the empty matrix and increases remarkably after filling. The comparison between the spectra of the filled and empty SBA-15 (Fig. 3 (b)) obtained after the equilibrium period, demonstrates the absence of systematic Bragg peak extinction and reveals that the intensities of the different Bragg orders are not affected in the same manner but are rather modulated. This proves that the approximations supporting Eq. (1) are no longer fulfilled. Generalizing the observations made for MCM-41, we can conclude without further analysis that the filling of the porous channels of SBA-15 with the contrast matching TBA/TOL mixture is complete but not homogeneous, which means that the concentration of the two types of molecules varies within the pore volume.

\section{B. Twin binary liquids}

The second strategy conducted to investigate the nature of the structuring of TBA-TOL mixtures in SBA-15 is the study of "twin binary liquids", which satisfy three specific features: First they have the same chemical mixture composition (i.e., same TBA-TOL volume fraction but different isotopic composition). Second they possess the same average total neutron scattering length density (i.e., they should exhibit the exact same diffraction patterns in the case of a homogeneous 
filling). Third, the binary mixture comprises only one visible deuterated component (TOL-(D) or TBA-(D)), while the other component is isotopically contrast matched with the matrix (TBA-(CM) or TOL-(CM)) and is thus "transparent" to neutrons. Only a unique chemical composition can fulfill all the conditions of twin symmetric mixtures. This composition is determined by the molecular scattering lengths and the liquid density. The volume fraction corresponding to this composition is TBA-TOL 43/57.

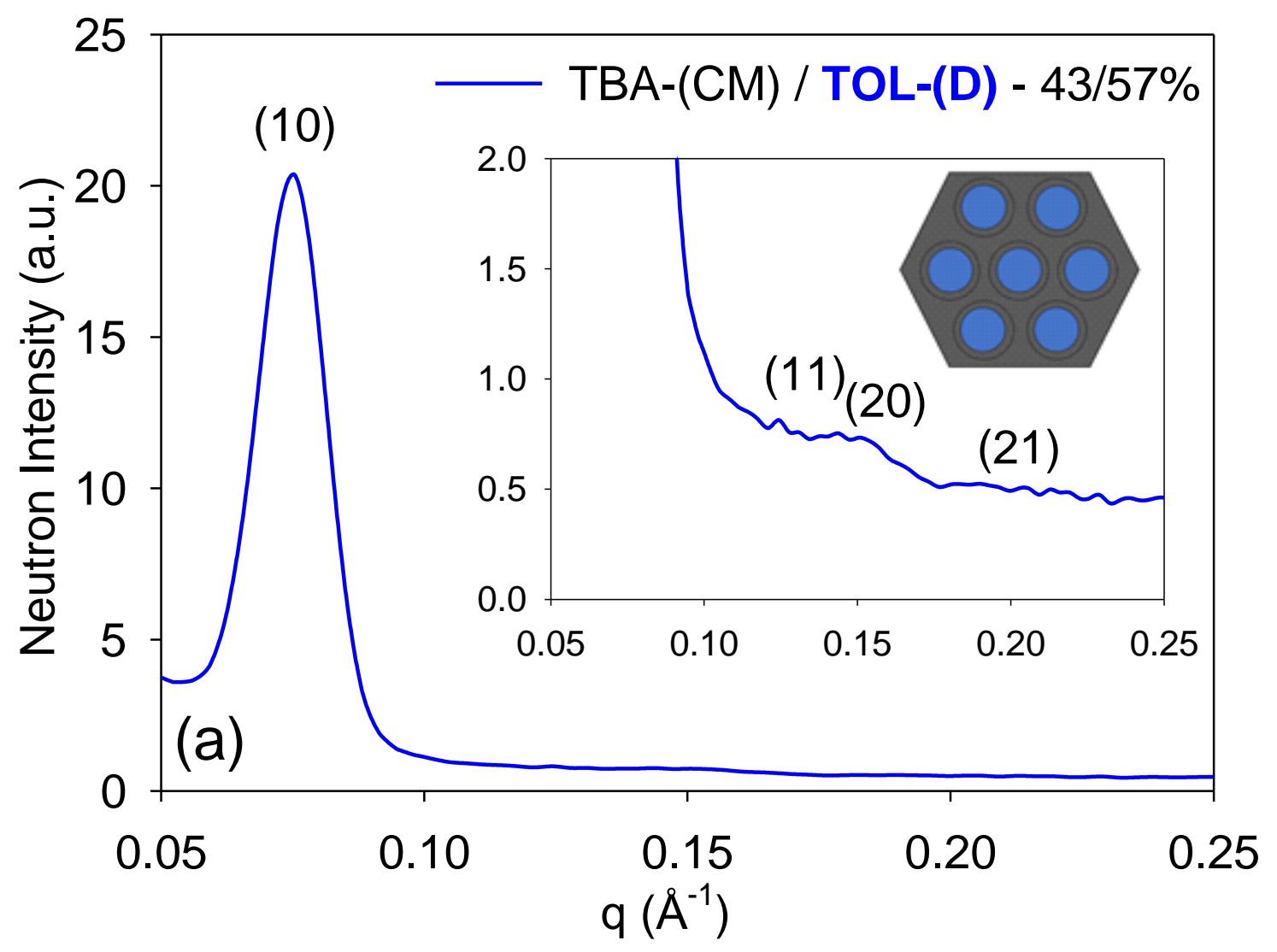




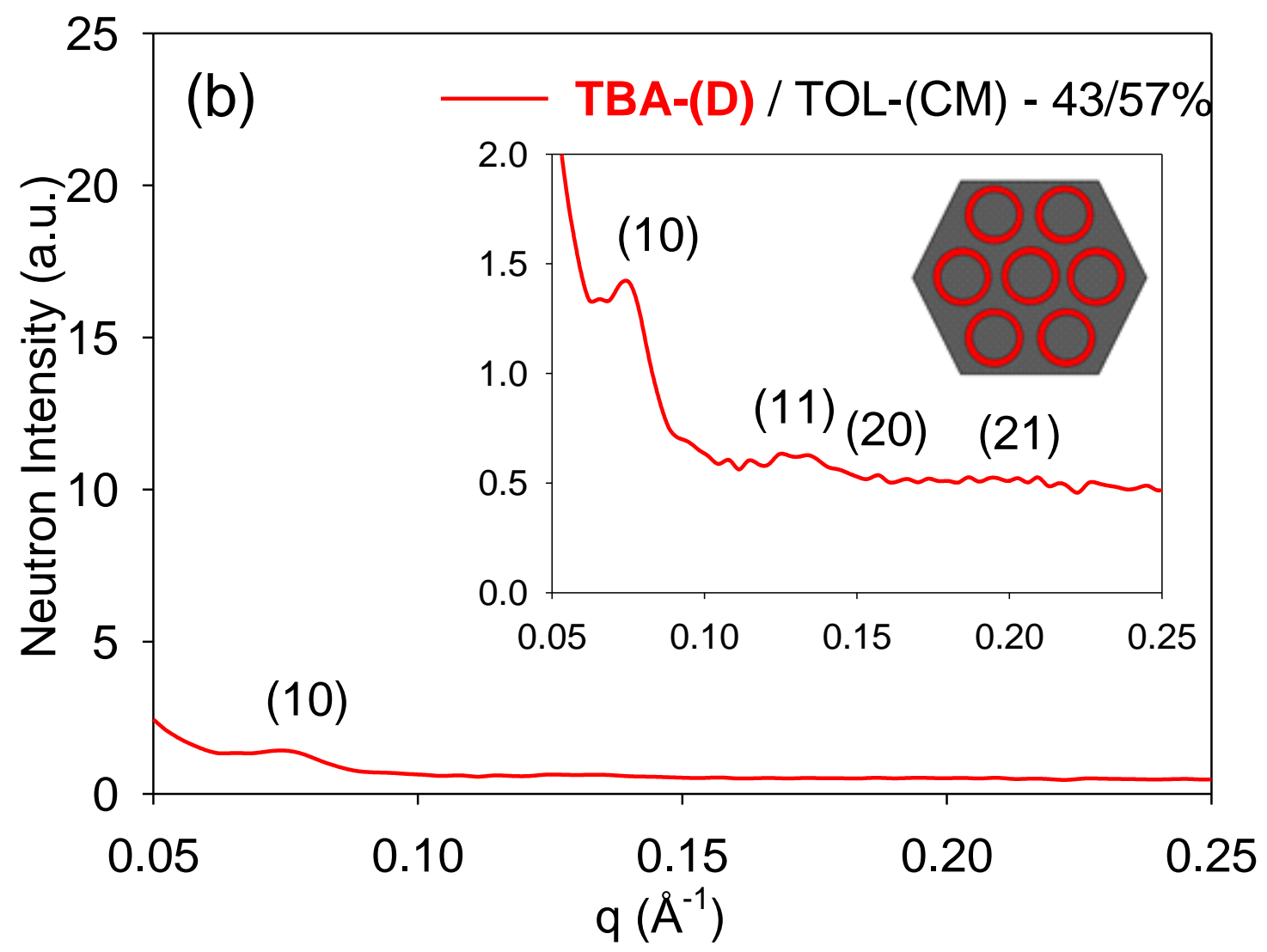

FIG. 4. Neutron diffraction patterns of SBA-15 filled with twin binary liquids made of an isotopic mixtures such that the average scattering length density is identical for the two liquids, the chemical composition is the same with a TBA-TOL volume fraction $43 / 57 \%$ and constituted from a fully deuterated component mixed with its contrast matching counterpart. (a) contrast matching TBA mixed with deuterated TOL and (b) deuterated TBA mixed with contrast matching TOL. Inset: sketch of a core-shell structure corresponding to the TBA@Surface model with TOL-(D) in blue, TBA-(D) in red, and silica, TOL-(CM) and TBA-(CM) in gray (see discussion part for details). 
The neutron structure factors of the two twin binary liquids are shown in Fig. 4 (a) and 4 (b). Although in the case of homogeneous filling their spectra should be identical, they exhibit remarkably different patterns with very distinct features supplying further evidence of concentration inhomogeneities across the SBA-15 pores. The scattered intensity of the TBA-(CM)

- TOL-(D) sample displays a very intense (10) and a relatively intense (20) Bragg peak. On the contrary, the structure factor of the TBA-(D) - TOL-(CM) twin system presents an almost total extinction. This different modulation of the Bragg peak intensities between the two twin samples clearly indicates a static inhomogeneous distribution of the concentration of the binary mixtures inside the pore. In addition, results from these twin mixtures are of prime interest to test structural models, and characterize the nature of the novel confined phase because the scattering intensity is solely sensitive to the deuterated component.

\section{DATA EVALUATION AND CORE-SHELL MODEL}

\section{A. Data evaluation}

The diffraction spectra comprise two different contributions: a continuous background and Bragg peaks located at the same $q_{h k}$ positions as the empty SBA-15, with $h$ and $k$ being the Miller indices of the triangular crystalline lattice. The background is mostly related to incoherent scattering possibly due to the presence of hydrogen from surface silanol groups and a low- $q$ diffuse scattering. The structural information related to the confined mixture could be extracted from the intensity of the Bragg peaks peaks after removal of the background $I_{B g}(q)$ interpolated with a pseudo Voigt function. Using the same procedure as ref. 19, the Bragg peaks were fitted by a weighted sum of unitary Gaussian functions $G_{h k}$ (cf. Fig. 5) centered at the Bragg peaks positions $q_{h k}$, thus reflecting the crystalline order of the triangular lattice and conforming to the Eq. (2) : 


$$
I_{f i t}(q)-I_{B g}(q)=\frac{1}{q^{2}} \sum_{h k} M_{h k} \tilde{I}\left(q_{h k}\right) G_{h k}\left(q-q_{h k}\right)
$$

$M_{h k}$ is the line multiplicity $\left(M_{h k}=6\right.$ for $(h 0)$ or $(h h)$ and 12 otherwise), and $\tilde{I}\left(q_{h k}\right)=$ $\int I\left(q_{h k}\right) q^{2} d q$, is the integrated Bragg peak intensity.

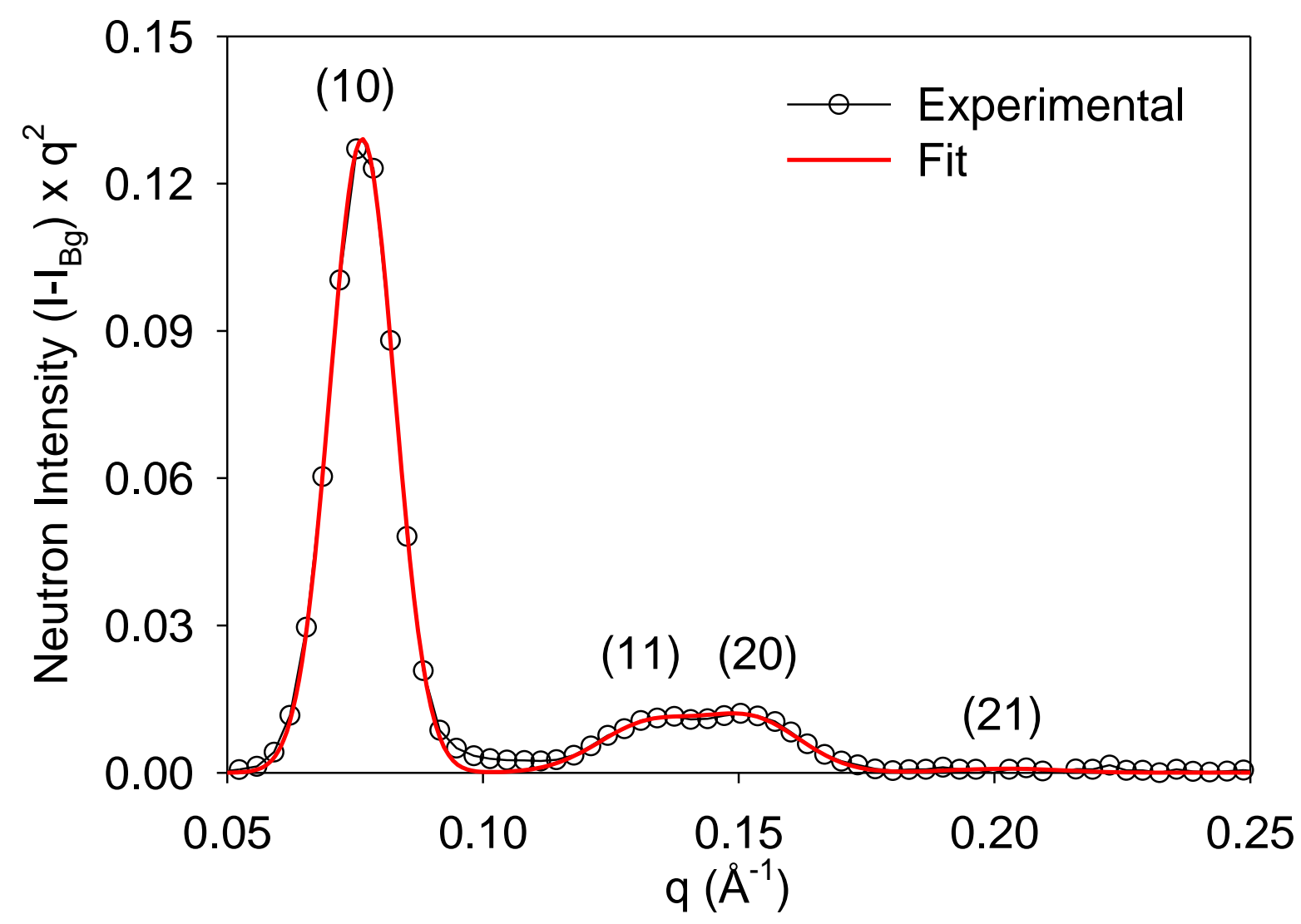

FIG. 5. Neutron diffraction intensity of empty SBA-15. The Bragg peaks are labelled by their Miller indexes and fitted by a sum of Gaussian functions (red line).

In this section, we attempt to interpret the variation of the measured integrated Bragg peak intensities $\tilde{I}\left(q_{h k}\right)$ in terms of various structural models similar to those used for the adsorption of 
fluids in mesoporous silicates. ${ }^{33-39}$ The neutron diffraction intensity of the SBA-15 materials, considered as a periodic triangular array of cylindrical pores is given by

$$
I(q)=K S(q)|F(q)|^{2}
$$

where $K$ is an instrumental normalization constant factor, $S(q)$ the powder averaged structure factor of the SBA-15 matrix and $F(q)$ the scattering amplitude of a single pore. $S(q)$ is a sum of Dirac functions $\delta$, while the form factor $F(q)$ comprises the essential structural information about the binary liquid confined in the porous channel according to Eq. (4).

$$
I(q)=\frac{K}{q^{2}} \sum_{h k} M_{h k} \delta\left(q-q_{h k}\right)|F(q)|^{2}
$$

Now comparing Eqs. (2) and (4) provides the principle of the data analysis, which consists in the evaluation of $|F(q)|^{2}$ for given models and the comparison of the predicted values $K\left|F\left(q_{h k}\right)\right|^{2}$ with the measured Bragg peak integrated intensities $\tilde{I}\left(q_{h k}\right)$.

\section{B. Core-shell model}

Now we detail different models, to describe the structure of the confined phases. It should be mentioned that SBA-15 being a 2D crystal, with a translational invariance along the direction of the channel axis, only the averaged structure of the confined liquids within a plane perpendicular to the channel axis matters. Moreover, considering the cylindrical symmetry of the pores, the structure of the confined liquid could be simply expressed in terms of a radial concentration profile

across the pore diameter. ${ }^{19}$ A concentric cylindrical core-shell model was chosen as the simplest way to describe the concentration profile in the pore: indeed it assumes only two different values of concentration. The static lattice distortions in the pore position from an ideal triangular lattice 
were accounted for by a Debye-Waller factor (DWF), $\mathrm{e}^{-\frac{\left.\mathrm{uu}^{2}\right\rangle \mathrm{Q}^{2}}{2}}$ as shown in Fig. 6 (a). ${ }^{19,33,35,36}$ Three levels of the scattering length density $\rho=n \bar{b}$ are considered, corresponding to the core $\left(\rho_{\text {core }}\right)$, shell $\left(\rho_{\text {shell }}\right)$, and silica $\left(\rho_{0}\right)$ as shown in Fig. $6(\mathrm{~b})$.
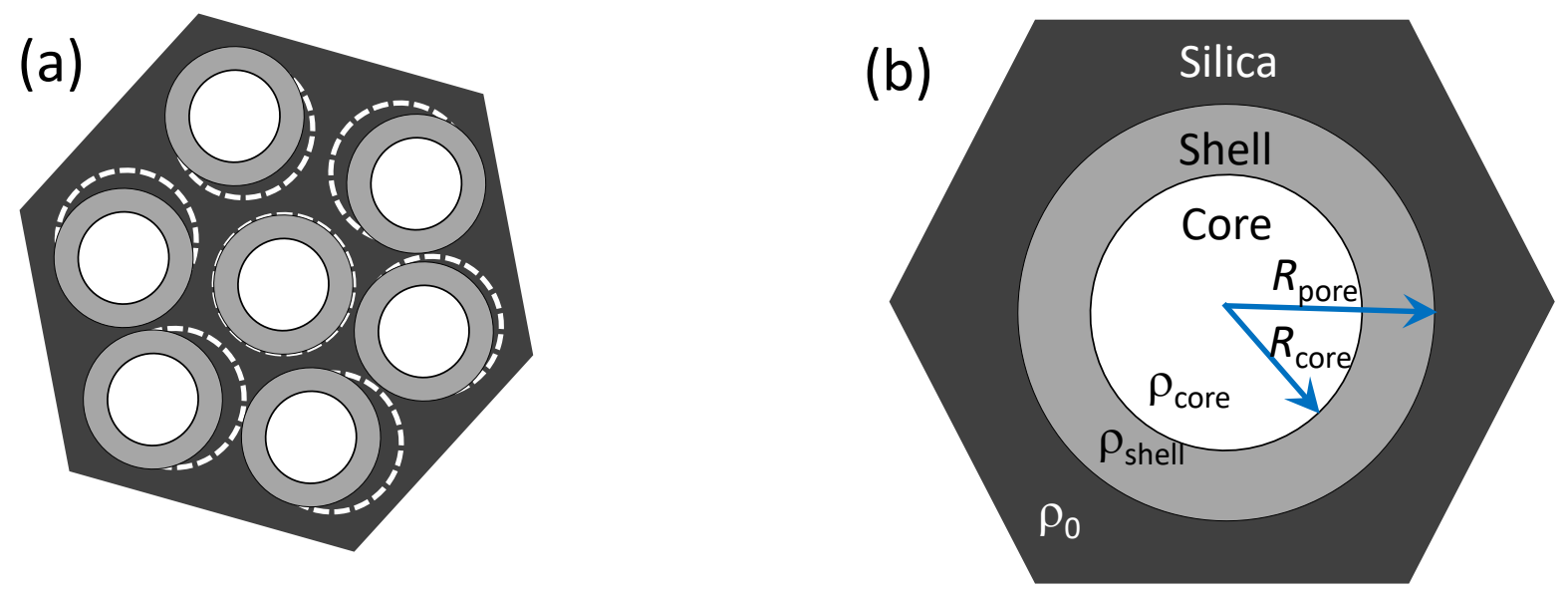

FIG. 6. Schematic representation of (a): the static random disorder of the pore positions from the ideal 2-D hexagonal lattice located by dashed lines. (b): Three density level core-shell model with a dense silica matrix $\left(\rho_{0}\right)$, shell $\left(\rho_{\text {shell }}\right)$, core $\left(\rho_{\text {core }}\right) . R_{\text {core }}$ and $R_{\text {pore }}$ represent the radii of the pore and of the internal core respectively.

The form factor of a single empty pore is deduced from the scattering amplitude of a cylindrical channel

$$
F^{E m p t y}(q)=\frac{\rho_{\text {Sio }_{2}} R_{\text {pore }}^{2} Z\left(q R_{\text {pore }}\right)}{\rho_{\mathrm{SiO}_{2}} R_{\text {pore }}^{2}}
$$

Where $\rho_{\mathrm{SiO}_{2}}$ is the coherent scattering length density of silica and $R_{\text {pore }}=4.15 \mathrm{~nm}$ is the pore radius determined from nitrogen adsorption based on the improved Kruk-Jaroniec-Sayari method. ${ }^{40}$ The function $Z$ is given by $Z(x)=2 \mathrm{~J}_{1}(x) / x$, with $\mathrm{J}_{1}$ being a first-order Bessel function. 
The value of the scattering length density of silica $\left(\rho_{\mathrm{SiO}_{2}}=34.6 \times 10^{9} \mathrm{~cm}^{-2}\right)$ was used in agreement with the contrast matching experiments illustrated in the previous part.

In the case of a filled matrix, we have proposed three possible concentration profiles:

1) A homogeneous filling of the pore by the mixture (single concentration profile). The resulting diffraction pattern is simply proportional to that of the empty SBA-15, with a different contrast factor in $F(q)$; (i.e., simple rescaling of the intensity with respect to the empty matrix).

$$
F(q)^{H o m o}=\frac{\left(\rho_{\text {liq }}-\rho_{\text {Sio }_{2}}\right)}{\rho_{S i o_{2}}} F(q)^{\text {Empty }}=\frac{\left(\rho_{\text {Liq }}-\rho_{\text {Sio }}\right) R_{\text {pore }}^{2} Z\left(q R_{\text {pore }}\right)}{\rho_{\text {Sio }} R_{\text {pore }}^{2}}
$$

2) A complete microphase separation of the two constituents of the binary mixture into a cylindrical core-shell structure where either TBA (later denoted TBA@surface model) or TOL (later denotedTBA@core model) is segregated at the surface of the pore, and the other component located in the core of the pore. The form factor for this core-shell structure is:

$$
F^{\text {Core-Shell }}(q)=\frac{1}{\rho_{\text {Sio } 2} R_{\text {pore }}^{2}} \sum_{i=1}^{2}\left(\rho_{i}-\rho_{(i-1)}\right) R_{i}^{2} Z\left(q R_{i}\right)
$$

Where $\rho_{0}=\rho_{\text {Sio }_{2}}, \rho_{1}=\rho_{\text {shell }}, \rho_{2}=\rho_{\text {core }}, R_{1}=R_{\text {pore }}$, and $R_{2}=R_{\text {core }}$. It is noteworthy that these models do not require any fitting parameter specific to the confined liquid and thus provide predictive results that could be directly compared to the experimental values. Indeed, the only two unknown values viz. the scaling factor $K$ and the $\operatorname{MSD}\left\langle u^{2}\right\rangle$, were extracted from the fits of the empty SBA-15. The core radius $R_{\text {core }}$ is simply determined from the mixture composition $x_{T B A}$. while $\rho_{\text {shell }}$ and $\rho_{\text {core }}$ are the scattering length densities of the pure liquids. In the case where 
TBA is forming the shell (TBA@surface model), the core size is $R_{\text {core }}=\sqrt{\left(1-x_{T B A}\right)} R_{\text {pore }}$. Otherwise, TBA forms the core and corresponds to: $R_{\text {core }}=\sqrt{x_{T B A}} R_{\text {pore }}$ (TBA@core model).

3) Monolayer at surface: this concentration profile is rather a variant of the TBA@surface coreshell model. It represents the case of the segregation of one molecular layer of TBA of thickness $e_{m o n o}$ at the pore surface while the remaining quantity of the TBA (not constituting the interfacial layer) mixes with the TOL inside the pore. In this TBA-layer@surface model, the core concentration of TBA is $x_{\text {core }}=\frac{x_{T B A}}{\alpha^{2}}+\left(1-\frac{1}{\alpha^{2}}\right)$ where $x_{T B A}$ is the average composition of the filling liquid and $\alpha=1-\frac{e_{\text {mono }}}{R_{\text {pore }}}$ with the thickness of the monolayer being set to $e_{\text {mono }}=0.6 \mathrm{~nm}$ (average molecular diameter of one TBA molecule obtained from a structural study on bulk $\left.\mathrm{TBA}^{41}\right)$. The core size is then simply defined as: $R_{\text {core }}=R_{\text {pore }}-e_{\text {mono }}$.

Some studies have shown that SBA-15 could be described by a variant model where the cylindrical pore structure exhibits corrugated pore walls and/or an interfacial microporous region denoted as a 'corona'. $33,35,36,37,38,39$ Therefore, a structural model accounting for a reduced density sponge-like corona around the pores was also evaluated. It is based on the assumption that the cylindrical mesopores that follow a perfect 2-D hexagonal lattice with a circular cross section are surrounded by a microporous corona with constant porosity, while dense silica forms the outer region. Since no significant difference was observed between the DWF and the corona models, we based our discussion solely on the DWF model, and the results obtained by this model were shown as supplementary material. It suggests that the observed variations of the diffraction patterns with the isotopic composition of the binary liquids are essentially determined by the microphase separation phenomenon with the formation of a core-shell structure and less sensitive to the details of the model used to represent the SBA-15. 


\section{DISCUSSION}

The strategy used in this study consists in comparing the scattering patterns displayed by silica nanotubes (SBA-15) filled by isotopically different liquid mixtures with models, without any fitted parameters. It means that all the parameters were first obtained independently from the filled samples : the pore radius was fixed to the value $R_{\text {pore }}=4.15 \mathrm{~nm}$ determined from nitrogen adsorption based on the improved KJS method, ${ }^{40}$ while the scaling factor $K$ and the mean square displacement (MSD) $\left\langle u^{2}\right\rangle$ parameters were estimated from fitting the form factor of the empty matrix as shown in Fig. 7. The obtained value of the MSD is $\left\langle u^{2}\right\rangle=0.5 \mathrm{~nm}^{2}$, which corresponds to average displacements in the order of $0.7 \mathrm{~nm}$, i.e. about $7 \%$ of the unit cell parameter $\left(\frac{\sqrt{\left\langle u^{2}\right\rangle}}{a}=7 \%\right)$ , is in a good agreement with previous studies. ${ }^{19,33}$ 


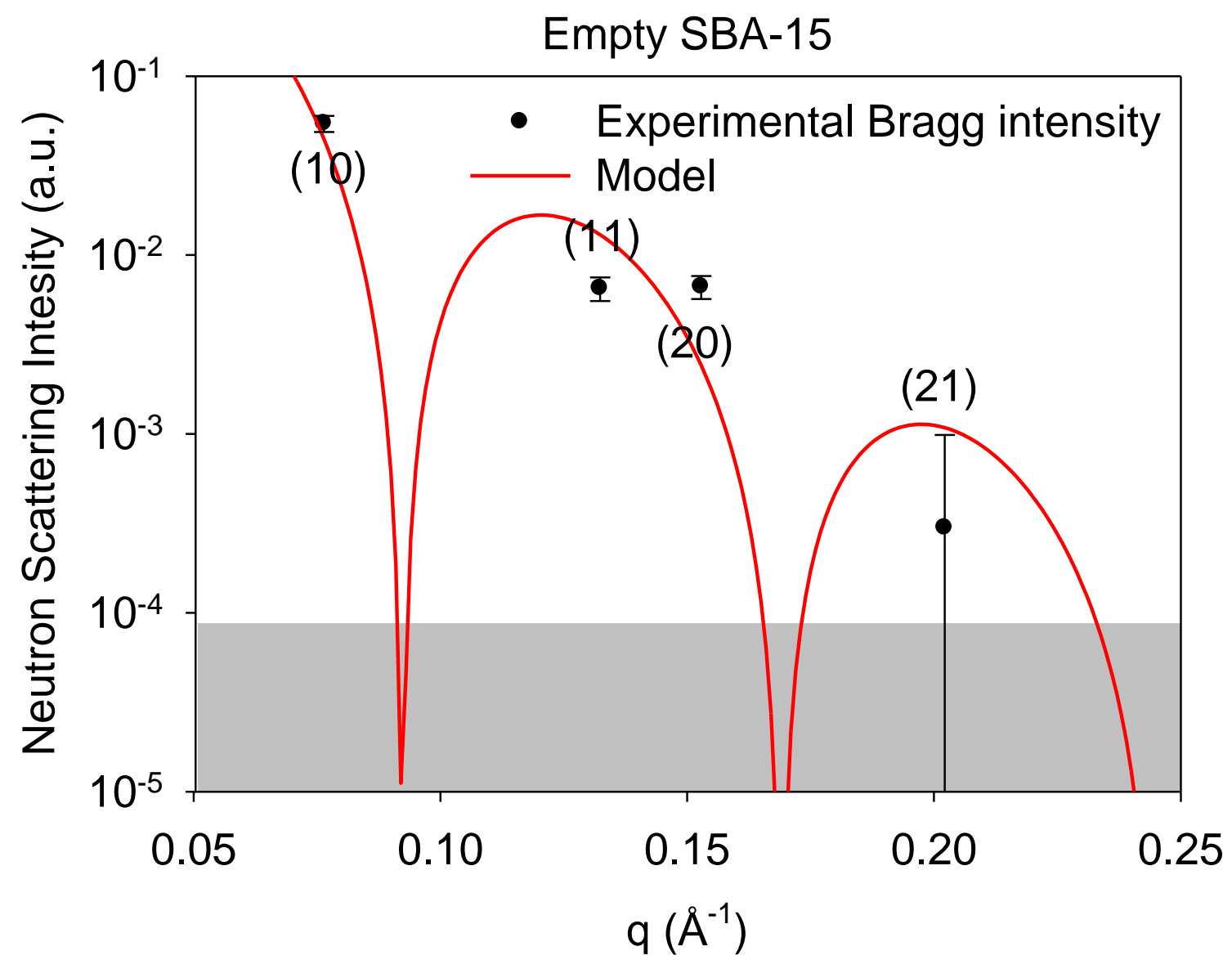

Fig. 7. Experimental value of the integrated intensity of the four Bragg peaks of the empty SBA15. The solid line is the result from the model fitting the scaling factor $K$ and the mean square displacement $\left\langle u^{2}\right\rangle$. The shading indicates the accuracy limit of the method.

The values of the core radius $R_{\text {core }}$ and the thickness of the shell $e_{\text {shell }}$ are fully determined from the sample composition and pore size. They are given in Table 1 for all the studied compositions and models. Depending on the model and on the mixture composition, these geometrical values span from one to five molecular sizes. It is interesting to note that for the specific composition TBA/TOL 30/70 the shell thickness $e_{\text {shell }}$ is equivalent to about one molecular layer. This makes 
bothTBA@surface and TBA-layer@surface models mathematically equivalent for this particular sample.

Table 1. Geometrical parameters of the two core-shell models and the monolayer model for the studied compositions. Note that these parameters were not fitted, but fixed by the composition of the mixture $\left(R_{\text {core }}\right)$ or retrieved from the study of the empty SBA-15 $\left(R_{\text {pore }}\right.$ and $\left.\left\langle u^{2}\right\rangle\right)$.

\begin{tabular}{|c|c|c|c|c|}
\hline Model & $R_{\text {core }}(\mathrm{nm})$ & $\begin{array}{l}\text { Shell thickness } \\
\boldsymbol{R}_{\text {pore }}-\boldsymbol{R}_{\text {core }}(\mathrm{nm})\end{array}$ & $R_{\text {pore }}(\mathrm{nm})$ & $\left\langle u^{2}\right\rangle\left(n m^{2}\right)$ \\
\hline \multicolumn{3}{|c|}{ Composition: $\mathrm{x}_{T B A}=0.37$} & \multirow{12}{*}{4.15} & \multirow{12}{*}{0.5} \\
\hline TBA@surface & 3.29 & 0.86 & & \\
\hline TBA@core & 2.52 & 1.63 & & \\
\hline TBA-layer@surface & 3.55 & 0.6 & & \\
\hline \multicolumn{3}{|c|}{ Composition: $\mathrm{x}_{T B A}=0.43$} & & \\
\hline TBA@surface & 3.13 & 1.02 & & \\
\hline TBA@core & 2.72 & 1.43 & & \\
\hline TBA-layer@surface & 3.55 & 0.6 & & \\
\hline \multicolumn{3}{|c|}{ Composition: $\mathrm{x}_{T B A}=0.3$} & & \\
\hline TBA@surface & 3.47 & 0.67 & & \\
\hline TBA@core & 2.27 & 1.87 & & \\
\hline TBA-layer@surface & 3.55 & 0.6 & & \\
\hline
\end{tabular}

We now test the ability of the computed form factors $|F(q)|^{2}$ to reproduce the extracted experimental intensities $\tilde{I}_{\mathrm{hk}}$ for different samples and different models. 
We first consider the TBA-(H)-TOL-(D) sample with the contrast matching composition (37/63). The predictions from the four different models are compared with the experimental values of the Bragg reflection intensity in Fig. 8. The radial profiles of the scattering length density across the pore are also sketched for the different models. Clearly, the model with homogeneous concentration profile fails to reproduce the experimental results, predicting an almost total extinction of the Bragg peaks for this almost perfect contrast matching composition. The predictions of the three other models all provide an explanation of the striking observation of nonvanishing Bragg peak intensities for this contrast-matching composition. They successfully predict a high intensity of the (10) Bragg peak. However, the TBA-layer@surface and TBA@surface models seem to be in better agreement than the TBA@core, especially for predicting the experimental intensities the second (11) Bragg peak.

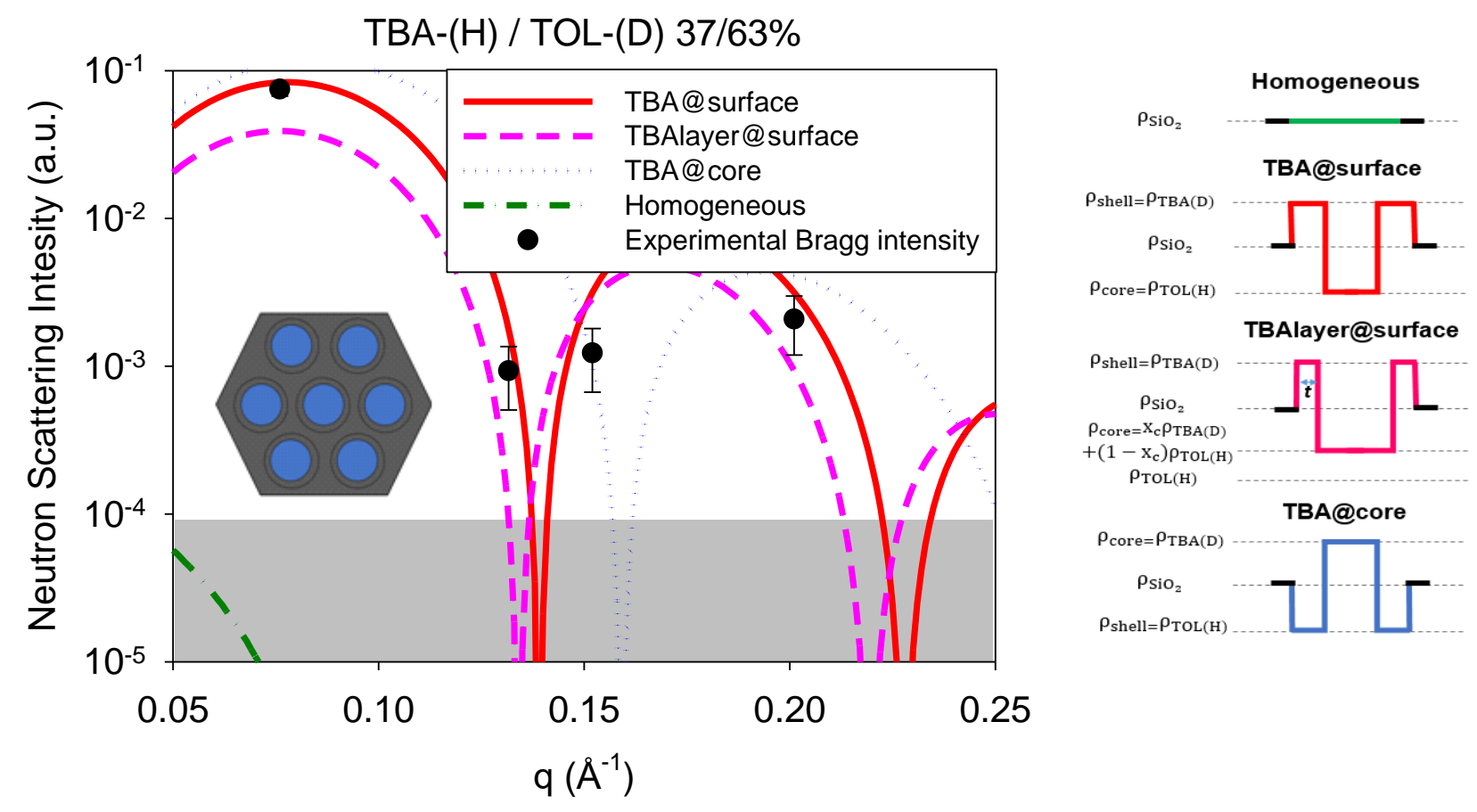

FIG. 8. Experimental value of the integrated intensity of the four Bragg peaks of the SBA-15 filled with a contrast matching binary mixture of hydrogenated Tert-butanol and deuterated Toluene in 
volume fraction $37 / 63 \%$. The lines are the predicted results from the model calculations according to Eq. (6) for four different cases: full mixing (homogeneous), microphase separated core-shell structure with TBA at the surface (TBA@Surface), microphase separated core-shell structure with TBA monolayer at the surface (TBA-layer@Surface) and microphase separated core-shell structure with TOL at the surface (TBA@Core). Right panel: Sketch of the scattering length density profile.

The indication that TBA rather than TOL stands at the pore surface is definitively confirmed by the other binary samples. To illustrate this point, we now consider the predictions for the two twin binary mixtures shown in Fig. 9. Again, the experimental trends are nicely predicted by the TBA@surface andTBA-layer@surface models as they could remarkably illustrate the unexpected difference in the Bragg peak intensities between the two twin samples. Both models have an equally good reproduction of the (10) Bragg reflection, which is very intense for the TBA-(CM) / TOL-(D) system (cf. Fig. 9 (a)), and weak for the TBA-(D) / TOL-(CM) sample (cf. Fig. 9 (b)). The intensities of the higher orders are also well-reproduced, providing an explanation for the inversion in the relative intensities of the (11) and (20) Bragg peaks between the two twin samples: On the one hand, the reduced intensity of the (11) Bragg peak in the TBA-(CM) / TOL-(D) (cf. Fig. 10 (a)) can be explained by the proximity of a cancellation point in the form factor. On the other hand, in the TBA-(D) / TOL-(CM) sample, the cancellation point in the form factor in both models is located at the same position as the (20) order peak, leading to its extinction (cf. Fig. 10 (b)). Generally, it is worth mentioning that, although the attained agreement remains semiquantitative, all the experimental systematic trends are successfully reproduced with the two simple models without any adjustment of their parameters. At variance, the TBA@core model fails to reproduce the observed experimental features. 

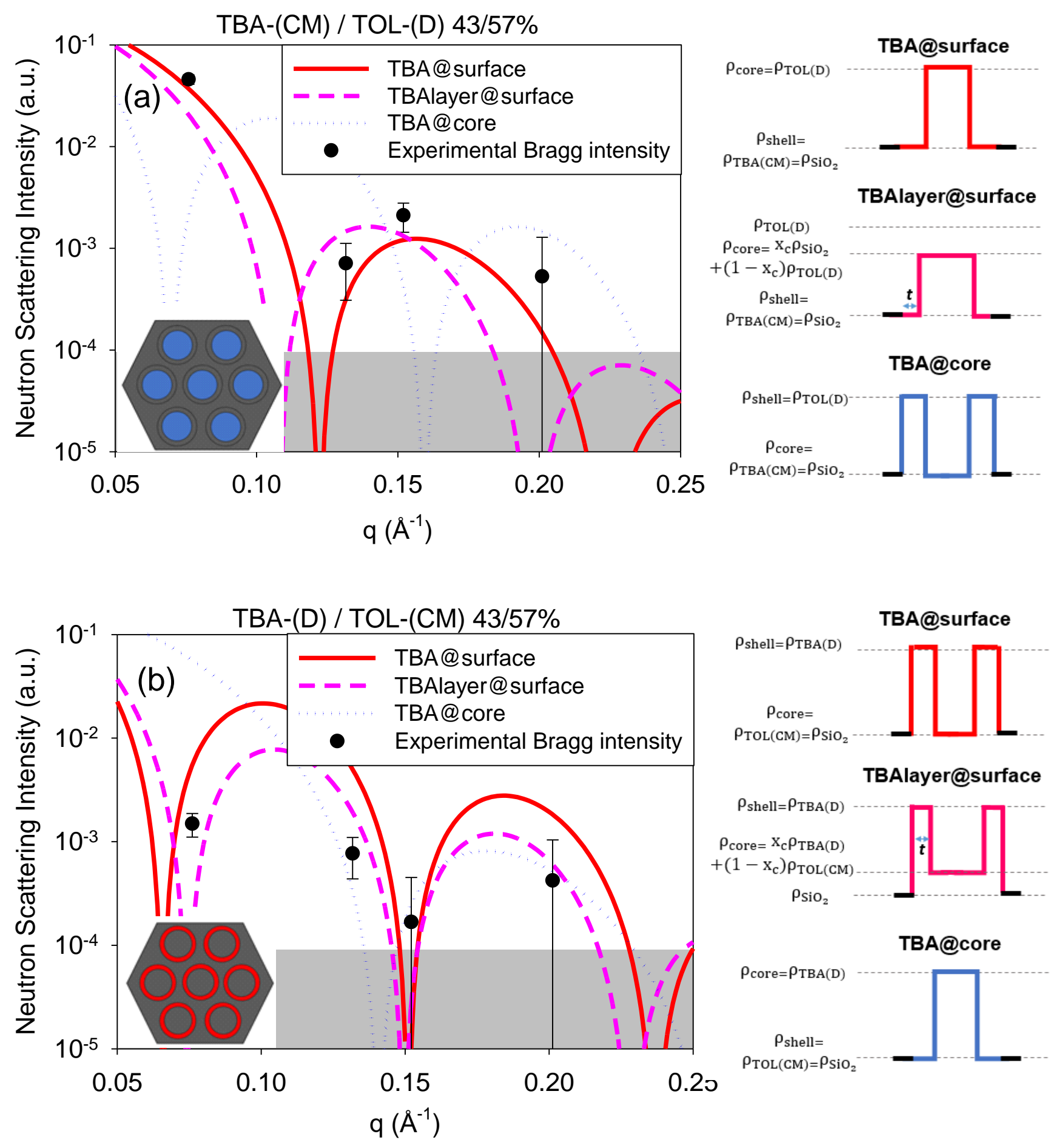

FIG. 9. Experimental value of the integrated intensity of the four Bragg peaks of the SBA-15 filled with symmetrical twin binary liquids having the same total average scattering length density, the same chemical composition (except isotopic differences) with a TBA/TOL volume fraction 
composition $43 / 57 \%$ and comprising a fully deuterated component mixed with its contrast matching counterpart. (A): contrast matching TBA mixed with deuterated TOL and (B): deuterated TBA mixed with contrast matching TOL. The solid lines are the predicted results from the model from the model calculations according to Eq. (6) for three different microphase separated coreshell structures with TBA at the surface (TBA@surface), TBA monolayer at the surface (TBAlayer@surface), and TOL at the surface (TBA@core). Right panel: Sketch of the scattering length density profile.

We now consider the TBA/TOL samples with a different value of the volume fraction, i.e. 30/70. In this case, an exceptional quality of the predictions is obtained with both the TBA@surface and TBA-layer@surface models, which are in quantitative agreement with the experimental intensities of the four Bragg peaks for both the TBA-(CM) / TOL-(D) sample (Fig. 10 (a)) and for the TBA(D) / TOL-(CM) sample (Fig. 10 (b)). More specifically, in the case of the TBA-(CM) / TOL-(D) sample, both models successfully predict the high intensities of the (10), (11) and (20) Bragg peaks and attribute the extinction of the (21) peak to the coincidence with a zero-value in the form factor (Fig. 11 (a)). The models also quantitatively reproduce the four Bragg reflections in the opposite TBA-(D) / TOL-(CM) sample (Fig. 11 (b)). It is also obvious that the predictions from the TBA@surface and TBA-layer@surface models are virtually undistinguishable. It was expected since these particular samples comprise the TBA volume fraction needed to form one molecular layer at the surface, thus making these two models mathematically equivalent (see Table 1). On the contrary, the opposite hypothesis that TOL would stand preferentially at the surface can be definitively ruled out. Indeed, the TBA@core model fits with none Bragg reflection and systematically fails to reproduce the experimental intensities. 

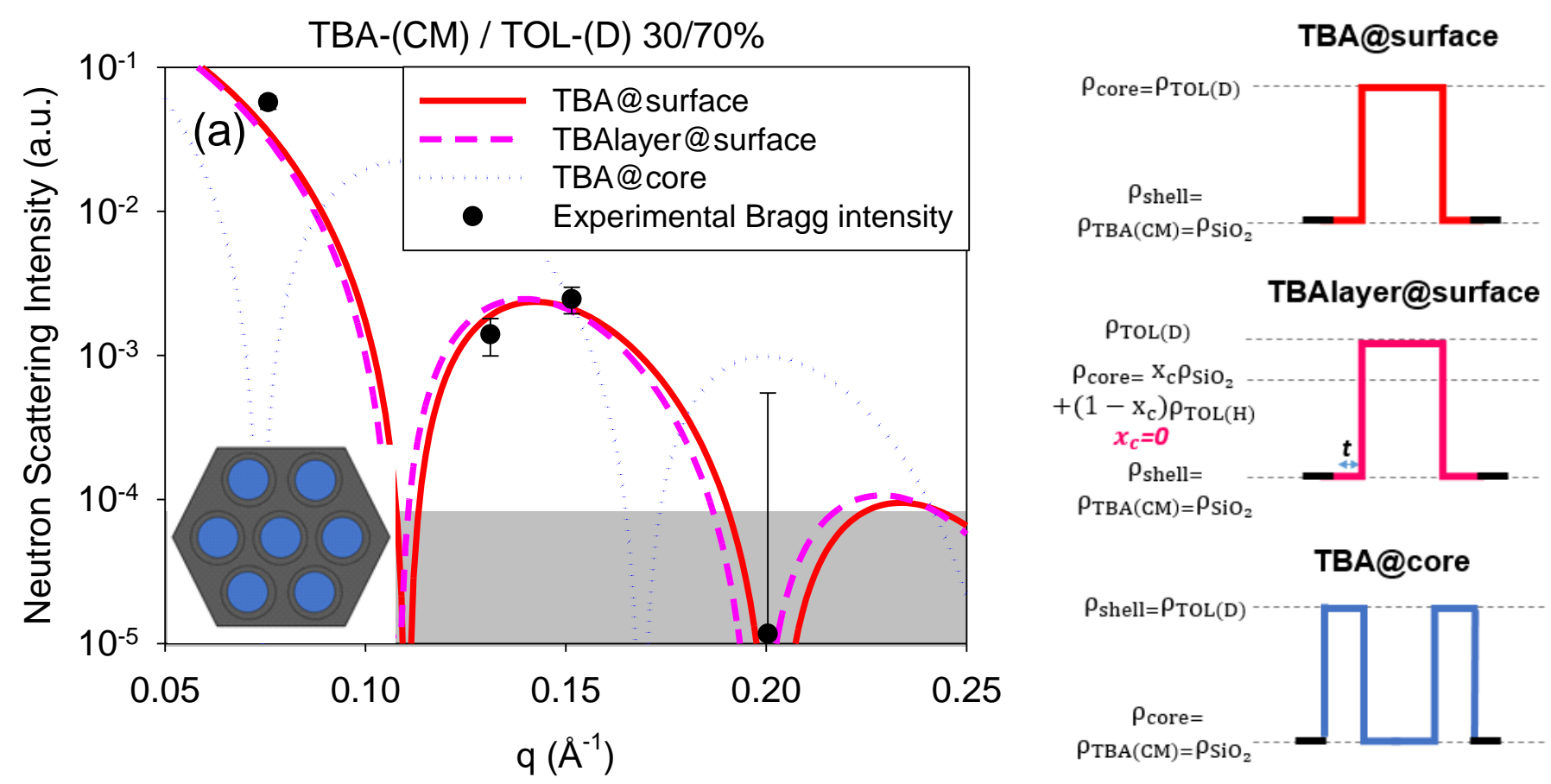

\section{TBAlayer@surface}
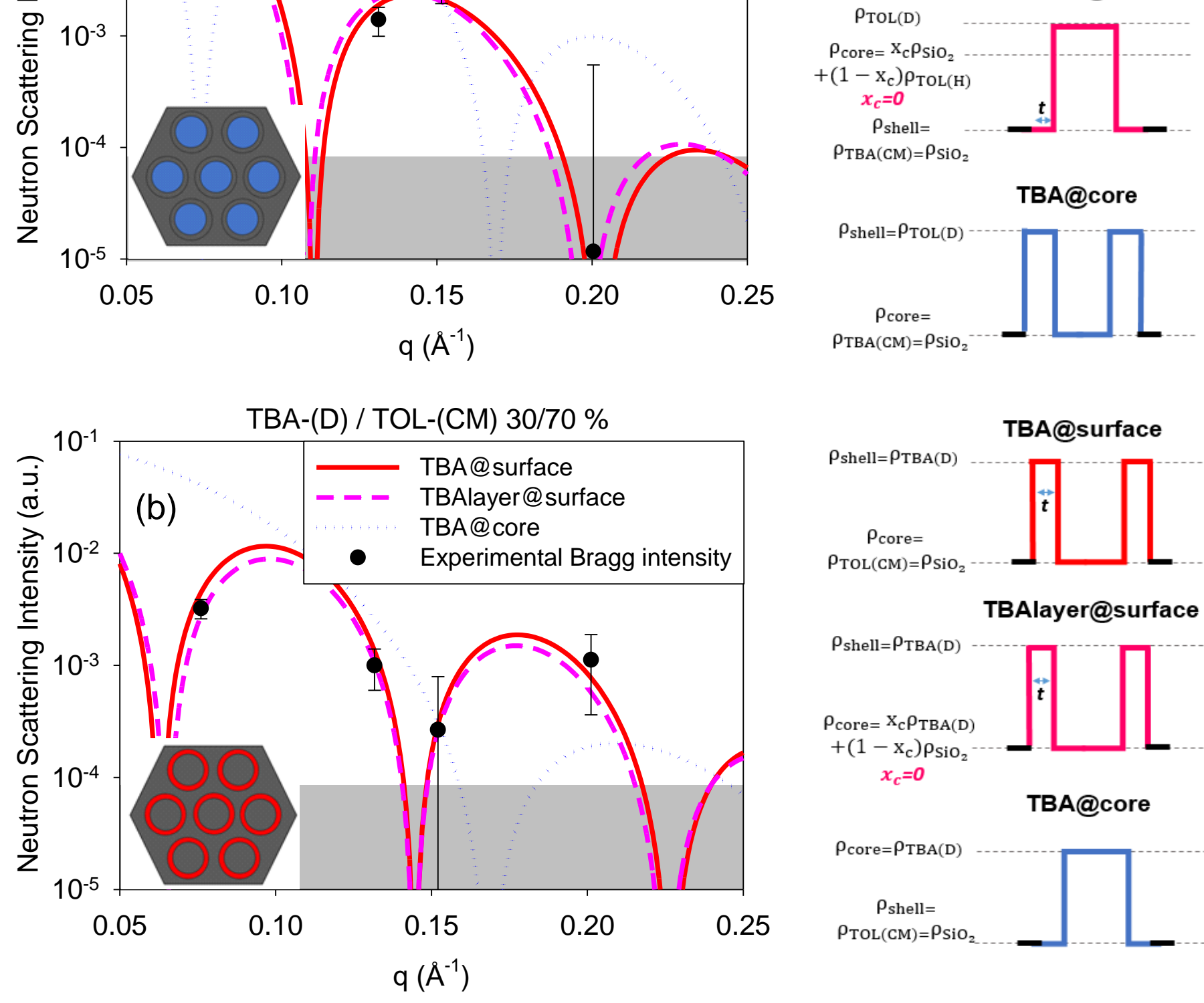

TBAlayer@surface
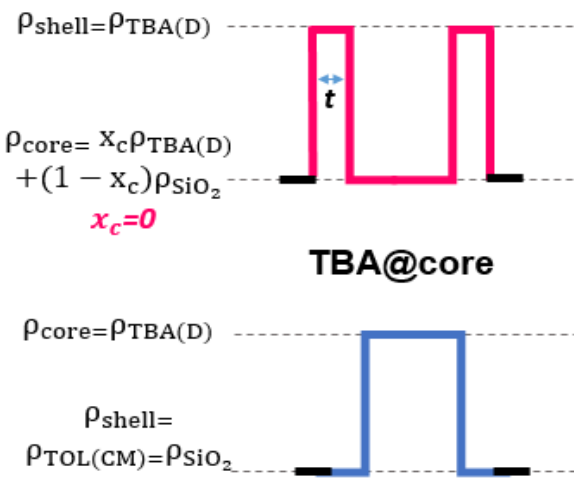

FIG. 10. Experimental value of the integrated intensity of the four Bragg peaks of SBA-15 filled with two binary liquids having the same chemical composition (except isotopic differences) with a TBA/TOL volume fraction composition $30 / 70 \%$ and comprising a fully deuterated component 
mixed with its contrast matching counterpart. (A): contrast matching TBA mixed with deuterated TOL and (B): deuterated TBA mixed with contrast matching TOL. The solid lines are the predicted results for three different models: microphase separated core-shell structures with TBA at the surface (TBA@surface), with one molecular TBA layer at the surface (TBA-layer@surface) and with TOL at the surface (TBA@core). Right panel: Sketch of the scattering length density profile.

The core-shell microphase separation is original with respect to the current knowledge of confined binary liquids because it concerns a fully miscible mixture. It is interesting to make a comparison with the existing literature on the liquid-liquid phase separation of binary liquids that already exhibit a miscibility gap in the bulk. ${ }^{42}$ According to molecular simulation studies of fluids, the thermodynamics and kinetics of demixing are deeply affected by confinement in infinite cylindrical pores. ${ }^{43,44,45}$ It was observed that the system does not phase separated completely but form long-lived metastable pluglike microdomains. A dramatic slowdown of the kinetics of phase separation is induced by the collective motions required for the condensation of two domains separated by a plug of the unlike molecules. This situation differs obviously from our observation, because plug-like structures would effectively lead to full extinction of the Bragg intensity for contrast matching mixtures. In the former studies, the formation of tube-like structures was unfavored by the choice of symmetrical interactions between the two types of molecules and the pore. On the contrary, surface-induced phase transition and the formation of an adsorbed film near the pore surface were observed for asymmetric binary systems. ${ }^{46}$ In line with our observations, it shows that the strength of the pore wall interaction with one of the two molecules plays on central role in the formation of surface-directed tube-like microstructures that were observed even at temperature above the two-phase coexistence region. 


\section{CONCLUSION}

There is currently a growing number of observations reporting on microphase separation induced under nanoporous confinement in liquid mixtures, which are under normal bulk conditions fully miscible. ${ }^{14,15,16,47,48}$ However direct evidence for such a phenomenon form structural investigations are scarce. ${ }^{19,20}$ In this article, we exploit both the unique properties of neutron scattering regarding isotopic labeling and the additional concentration parameter offered for mixtures to carefully design selective contrast conditions which undoubtly demonstrate the spontaneous microphase separation of different TBA-TOL miscible liquid mixtures under confinement in the nanochannels of SBA-15 mesoporous materials $(D=8.3 \mathrm{~nm})$.

These conclusions were obtained from the analysis of the diffraction pattern produced by filled and unfilled SBA-15 pore lattices, which can be directly related to the radial concentration profile of the confined mixture across the channel. In that aspect, we implemented simple core-shell models having the advantage of being fully predictive, with parameters already determined and fixed by the study of the empty matrix. We derived different variant of the model, assuming either the complete phase separation of the two constituents (TBA@surface and TBA@core), or the formation of one molecular layer (TBA-layer@surface). We also accounted for the irregularity of the SBA-15 porous geometry, in line with the existing literature, introducing either an effective Debye-Waller factor or a microporous corona. Regardless of their simplicity, all the core-shell models predictions that assume the preferential segregation of TBA at the pore surface surrounding a TOL-rich core nicely reproduce all the experimental trends. They explain the variation of the Bragg intensity at the semi-quantitative, and most often at the quantitative level, depending on the system. 
The obtained concentric core-shell tubular structure corresponds to the segregation of alcohols in an interfacial TBA-rich region of about one to three molecular sizes, surrounding a TOL rich liquid tube. This result extends the observation firstly reported for the same liquid mixture confined in the MCM-41 materials with smaller pores $(D=3.65 \mathrm{~nm}) \cdot{ }^{19} \mathrm{In}$ addition, it shows that the microphase separation, which was first exemplified for binary mixtures with a TBA volume $x=0.51$ and 0.43 , can be observed for a broader range of compositions, at least down to $x$ $=0.3$. This indicates that the original nano-demixing phenomenon can be extended to different confining matrices with larger pore sizes and different mixture compositions, answering the questions raised in the introduction part of the article about the general character of the concept of microphase-separation.

However the overall results obtained for SBA-15 and MCM-41 mesoporous materials remain limited to hydrophilic matrices so far. It would be certainly interesting to vary the nature of interfacial interaction and the pore surface, using hydrophobic matrices in order to assess different anticipated core-shell structures. Another approach would be changing the nature of the liquids to test the applicability of this nanostructuring on mixtures with different structural properties (aromaticity, size, hydrophobicity...). It is noteworthy that TBA spontaneously forms supermolecular cyclic clusters in the bulk state, although TBA-TOL mixtures are homogeneous at the macroscopic scale. ${ }^{21}$ It would be of great interest to assess how such self-assemblies, which are observed in the bulk mixtures could influence the formation of core-shell structures in the confined state. For that purpose, one should also consider the case of smaller alcohol molecules, such as methanol or ethanol, in order to investigate different chain-like H-bonded structures. Ongoing structural studies will address these issues making use of the benefits of the crystallinity 
of the arrangement mesoporous channels and the selective isotopic sensitivity of the neutron interaction in order to achieve a unique level of description.

Finally, our study suggests that microphase separation, as a new type of nanostructure results from the concomitant effects of specific surface interactions and spatial confinement. The modulation of both elements could provide new opportunities to control supermolecular assembling of complex mixtures.

\section{ACKNOWLEDGMENTS}

The experiments were performed in the frame of the $\mathrm{PhD}$ project of $\mathrm{R}$. Mhanna who acknowledges funding by the Institute Laue-Langevin and the Brittany Region (ARED 7784 / NanoBina). Support from Europe (FEDER) and Rennes Metropole is expressly acknowledged. We thank Cristelle MÉRIADEC (Institut de Physique de Rennes) and Odile MERDRIGNAC (Institut des Sciences Chimiques de Rennes) for their assistance with the X-ray diffraction and Nitrogen isotherms experiments respectively for the characterization of the SBA-15 materials.

\section{SUPPLEMENTARY MATERIALS}

We also implemented the same core-shell model with an additional (corona) layer accounting for the wall structure, which was modeled by corrugated pore walls comprising an interfacial microporous region denoted as a 'corona' with a reduced silica density. The predictions from this variant model are compared with the predictions from the model detailed in the manuscript that uses a Debye Waller factor instead. Despite slight quantitative differences, the conclusions derived from both models are in full agreement, and demonstrate the existence of a micro-phase-separated 
tubular structure with the segregation of tert-butanol that forms a layer at the pore surface surrounding a toluene-rich core. 


\section{REFERENCES}

1. M. Alcoutlabi and G. B. McKenna, Journal of Physics-Condensed Matter 17 (15), R461R524 (2005).

2. C. Alba-Simionesco, G. Dosseh, E. Dumont, B. Frick, B. Geil, D. Morineau, V. Teboul and Y. Xia, The European Physical Journal E 12 (1), 19-28 (2003).

3. D. Morineau and C. Alba-Simionesco, The Journal of Physical Chemistry Letters 1 (7), $1155-1159$ (2010).

4. C. Alba-Simionesco, B. Coasne, G. Dosseh, G. Dudziak, K. Gubbins, R. Radhakrishnan and M. Sliwinska-Bartkowiak, Journal of Physics: Condensed Matter 18 (6), R15 (2006).

5. G. Chahine, A. V. Kityk, K. Knorr, R. Lefort, M. Guendouz, D. Morineau and P. Huber, Physical Review E 81 (3), 031703 (2010).

6. C. Cerclier, M. Ndao, R. Busselez, R. Lefort, E. Grelet, P. Huber, A. Kityk, L. Noirez, A. Schonhals and D. Morineau, Journal of Physical Chemistry C 116 (35), 18990-18998 (2012).

7. D. Morineau and C. Alba-Simionesco, The Journal of Chemical Physics 118 (20), 93899400 (2003).

8. D. Morineau, Y. Xia and C. Alba-Simionesco, The Journal of Chemical Physics 117 (19), 8966-8972 (2002).

9. A. V. Kityk, M. Wolff, K. Knorr, D. Morineau, R. Lefort and P. Huber, Physical Review Letters 101 (18), 187801 (2008).

10. R. Guégan, D. Morineau and C. Alba-Simionesco, Chemical Physics 317 (2-3), 236-244 (2005). 
11. A. Ghoufi, I. Hureau, R. Lefort and D. Morineau, The Journal of Physical Chemistry C 115 (36), 17761-17767 (2011).

12. R. Lefort, F. Jean, L. Noirez, M. Ndao, C. Cerclier and D. Morineau, Applied Physics Letters 105 (20), 203106 (2014).

13. A. Ghoufi, I. Hureau, D. Morineau, R. Renou and A. Szymczyk, Journal of Physical Chemistry C 117 (29), 15203-15212 (2013).

14. K. Elamin, H. Jansson, S. Kittaka and J. Swenson, Physical Chemistry Chemical Physics 15 (42), 18437-18444 (2013).

15. K. Elamin, H. Jansson and J. Swenson, Physical Chemistry Chemical Physics 17 (19), 12978-12987 (2015).

16. J. Swenson, K. Elamin, G. Chen, W. Lohstroh and V. G. Sakai, Journal of Chemical Physics 141 (21), 214501 (2014).

17. A. Lerbret, G. Lelong, P. E. Mason, M. L. Saboungi and J. W. Brady, Journal of Physical Chemistry B 115 (5), 910-918 (2011).

18. X. Y. Guo, T. Watermann and D. Sebastiani, Journal of Physical Chemistry B 118 (34), 10207-10213 (2014).

19. A. R. A. Hamid, R. Mhanna, R. Lefort, A. Ghoufi, C. Alba-Simionesco, B. Frick and D. Morineau, Journal of Physical Chemistry C 120 (17), 9245-9252 (2016).

20. A. R. A. Hamid, R. Mhanna, P. Catrou, Y. Bulteau, R. Lefort and D. Morineau, Journal of Physical Chemistry C 120 (20), 11049-11053 (2016).

21. R. Mhanna, R. Lefort, L. Noirez and D. Morineau, Journal of Molecular Liquids 218, 198-207 (2016). 
22. S. Dutta, R. Lefort, D. Morineau, R. Mhanna, O. Merdrignac-Conanec, A. Saint-Jalmes and T. Leclercq, Physical Chemistry Chemical Physics 18 (35), 24361-24369 (2016).

23. M. Mizukami, M. Moteki and K. Kurihara, Journal of the American Chemical Society 124 (43), 12889-12897 (2002).

24. M. Mizukami and K. Kurihara, Australian Journal of Chemistry 56 (10), 1071-1080 (2003).

25. M. Mizukami, Y. Nakagawa and K. Kurihara, Langmuir 21 (21), 9402-9405 (2005).

26. M. Mizukami and K. Kurihara, in Adsorption and Nanostructures, edited by I. Dekany (2002), Vol. 117, pp. 13-17.

27. M. Mizukami and K. Kurihara, Chemistry Letters (3), 256-257 (2000).

28. D. Y. Zhao, Q. S. Huo, J. L. Feng, B. F. Chmelka and G. D. Stucky, Journal of the American Chemical Society 120 (24), 6024-6036 (1998).

29. N. Brodie-Linder, G. Dosseh, C. Alba-Simonesco, F. Audonnet and M. Imperor-Clerc, Materials Chemistry and Physics 108 (1), 73-81 (2008).

30. D. Morineau, Y. Xia and C. Alba-Simionesco, Journal of Chemical Physics 117 (19), 8966-8972 (2002).

31. C. Balzer, R. Morak, M. Erko, C. Triantafillidis, N. Husing, G. Reichenauer and O. Paris, Zeitschrift Fur Physikalische Chemie-International Journal of Research in Physical Chemistry \& Chemical Physics 229 (7-8), 1189-1209 (2015).

32. G. Y. Gor, L. Bertinetti, N. Bernstein, T. Hofmann, P. Fratzl and P. Huber, Applied Physics Letters 106 (26), 261901 (2015).

33. M. Imperor-Clerc, P. Davidson and A. Davidson, Journal of the American Chemical Society 122 (48), 11925-11933 (2000). 
34. T. Hofmann, D. Wallacher, P. Huber, R. Birringer, K. Knorr, A. Schreiber and G. H. Findenegg, Physical Review B 72 (6), 064122 (2005).

35. G. A. Zickler, S. Jaehnert, W. Wagermaier, S. S. Funari, G. H. Findenegg and O. Paris, Physical Review B 73 (18), 184109 (2006).

36. S. Jaehnert, D. Mueter, J. Prass, G. A. Zickler, O. Paris and G. H. Findenegg, Journal of Physical Chemistry C 113 (34), 15201-15210 (2009).

37. D. Mueter, S. Jaehnert, J. W. C. Dunlop, G. H. Findenegg and O. Paris, Journal of Physical Chemistry C 113 (34), 15211-15217 (2009).

38. G. H. Findenegg, S. Jaehnert, D. Mueter, J. Prass and O. Paris, Physical Chemistry Chemical Physics 12 (26), 7211-7220 (2010).

39. R. A. Pollock, B. R. Walsh, J. Fry, I. T. Ghampson, Y. B. Melnichenko, H. Kaiser, R. Pynn, W. J. DeSisto, M. C. Wheeler and B. G. Frederick, Chemistry of Materials 23 (17), 38283840 (2011).

40. M. Jaroniec and L. A. Solovyov, Langmuir 22 (16), 6757-6760 (2006).

41. A. Perera, F. Sokolic and L. Zoranic, Physical Review E 75 (6), R060502 (2007).

42. A. J. Liu, D. J. Durian, E. Herbolzheimer and S. A. Safran, Physical Review Letters 65 (15), 1897-1900 (1990).

43. L. D. Gelb and K. E. Gubbins, Physical Review E 56 (3), 3185-3196 (1997).

44. L. D. Gelb and K. E. Gubbins, Physical Review E 55 (2), R1290-R1293 (1997).

45. Z. P. Zhang and A. Chakrabarti, Physical Review E 50 (6), R4290-R4293 (1994).

46. D. Woywod, S. Schemmel, G. Rother, G. H. Findenegg and M. Schoen, Journal of Chemical Physics 122 (12), 124510 (2005). 
47. A. Lerbret, G. Lelong, P. E. Mason, M.-L. Saboungi and J. W. Brady, Journal of Physical Chemistry B 115 (5), 910-918 (2011).

48. X.-Y. Guo, T. Watermann and D. Sebastiani, Journal of Physical Chemistry B 118 (34), 10207-10213 (2014). 


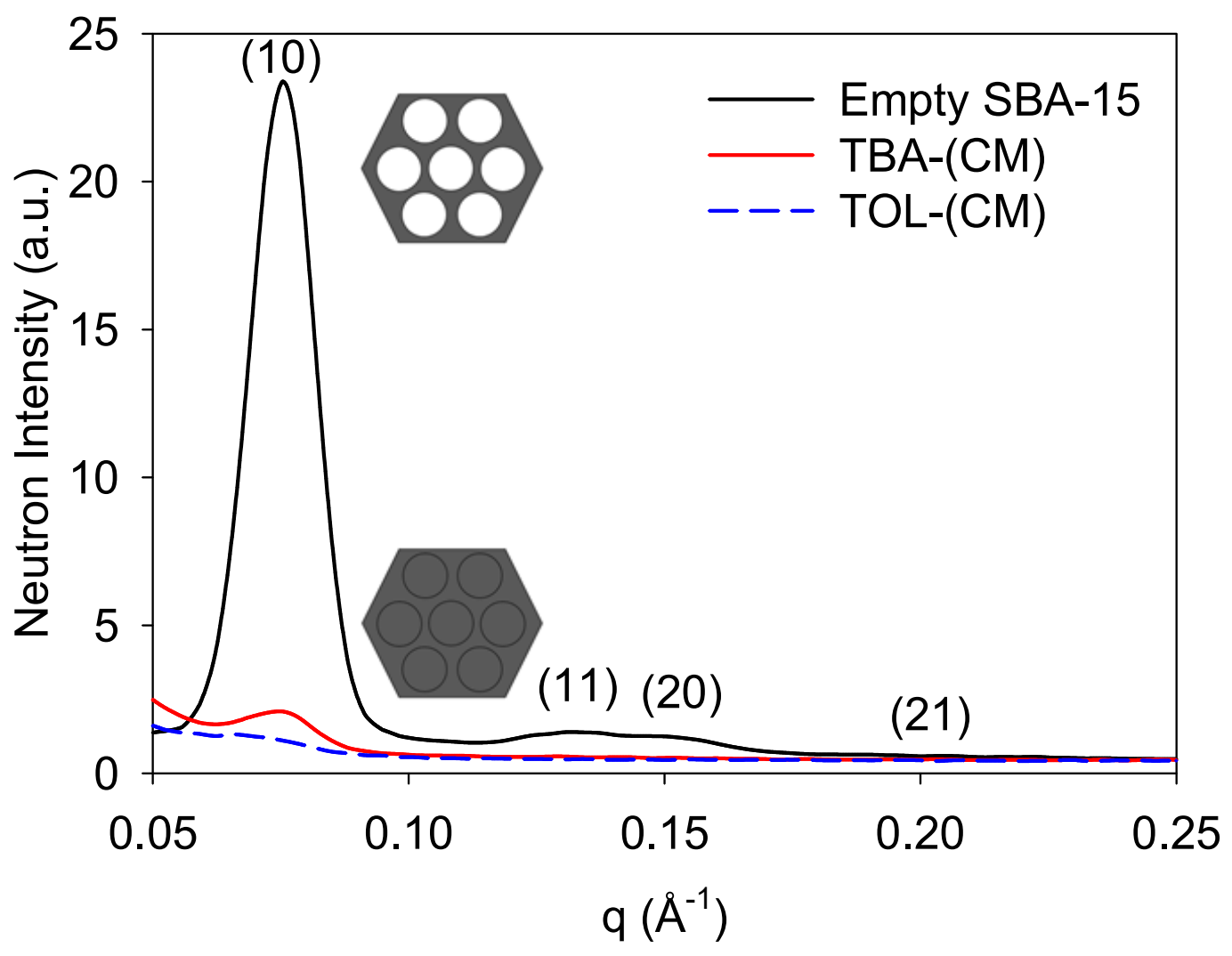




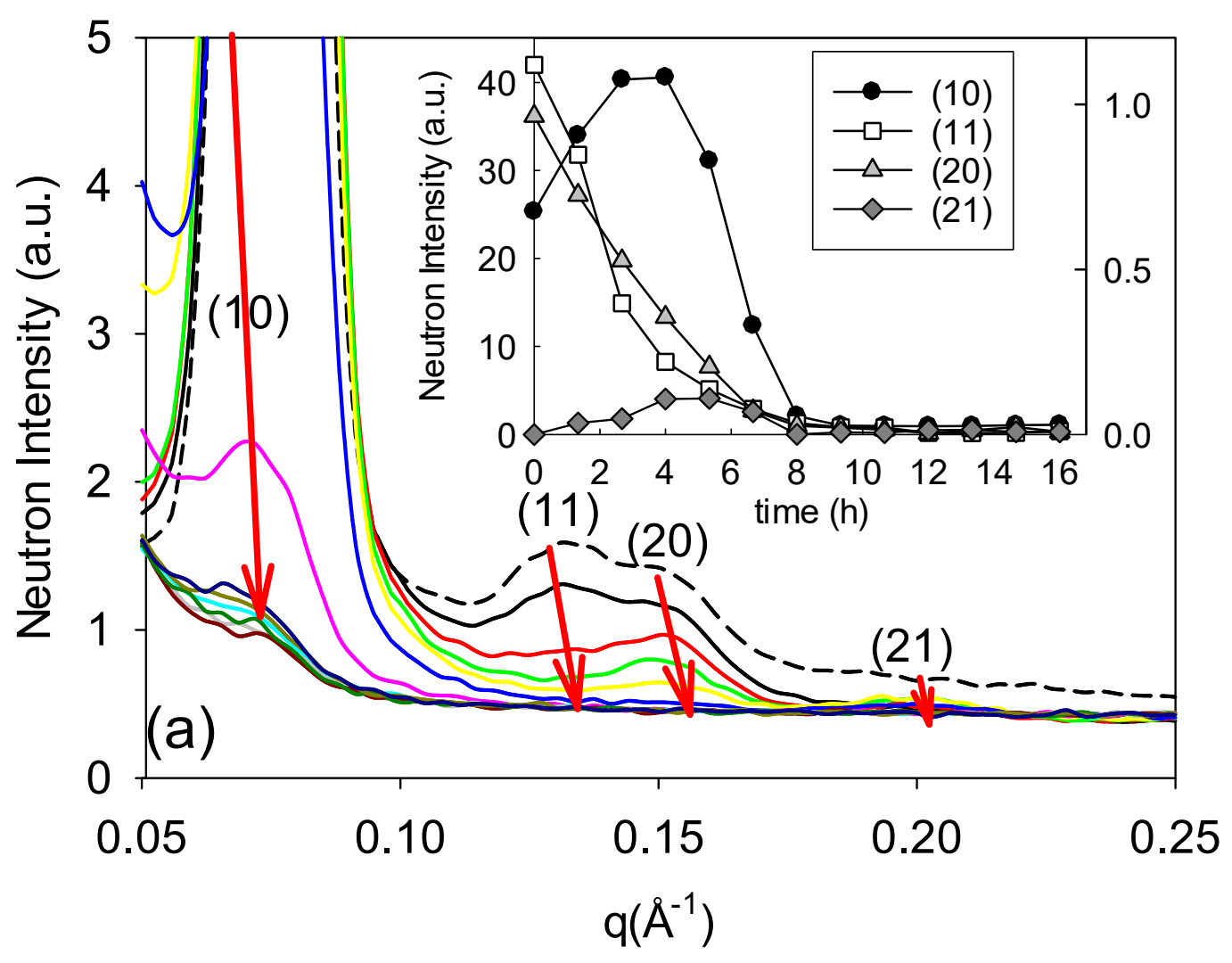




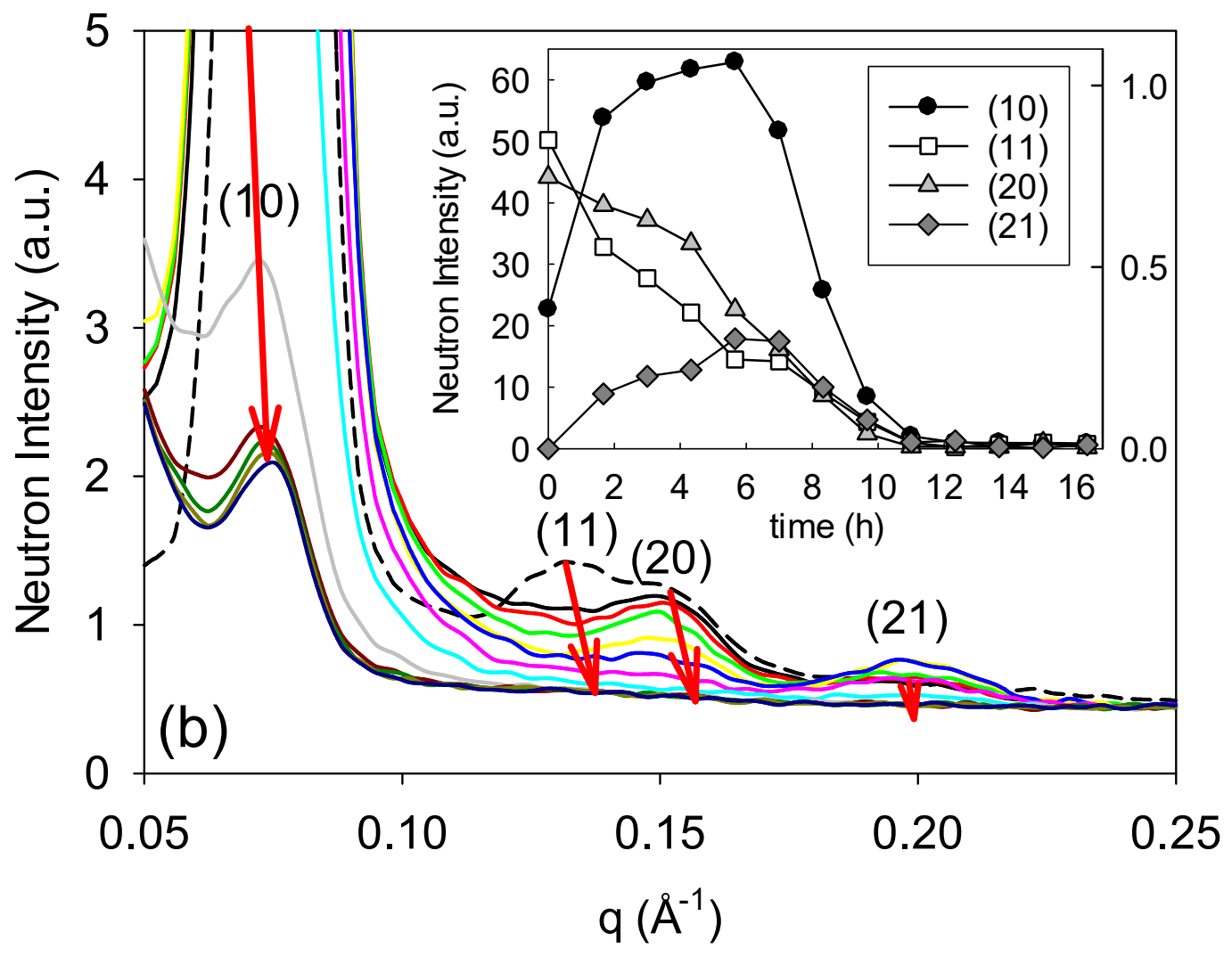




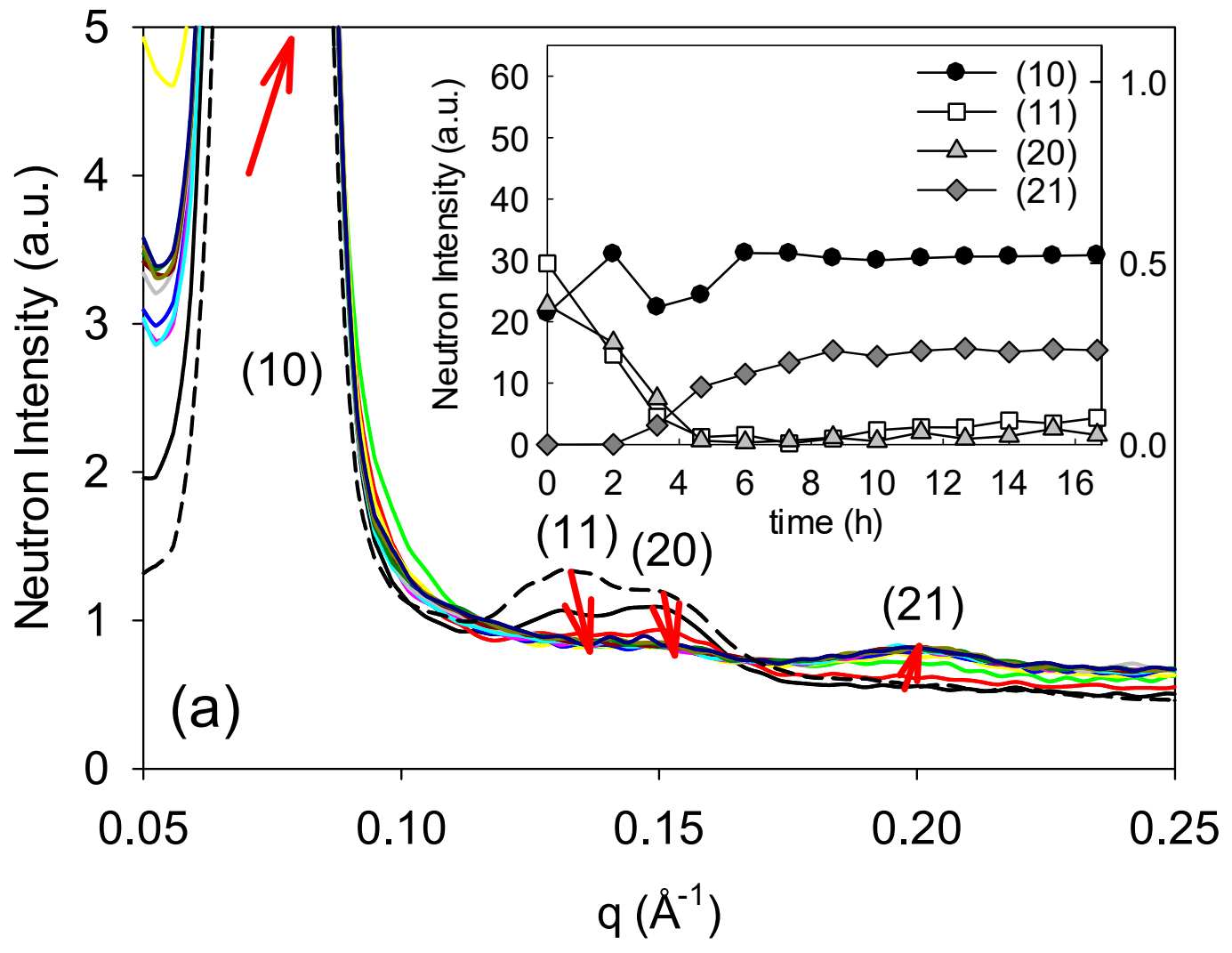




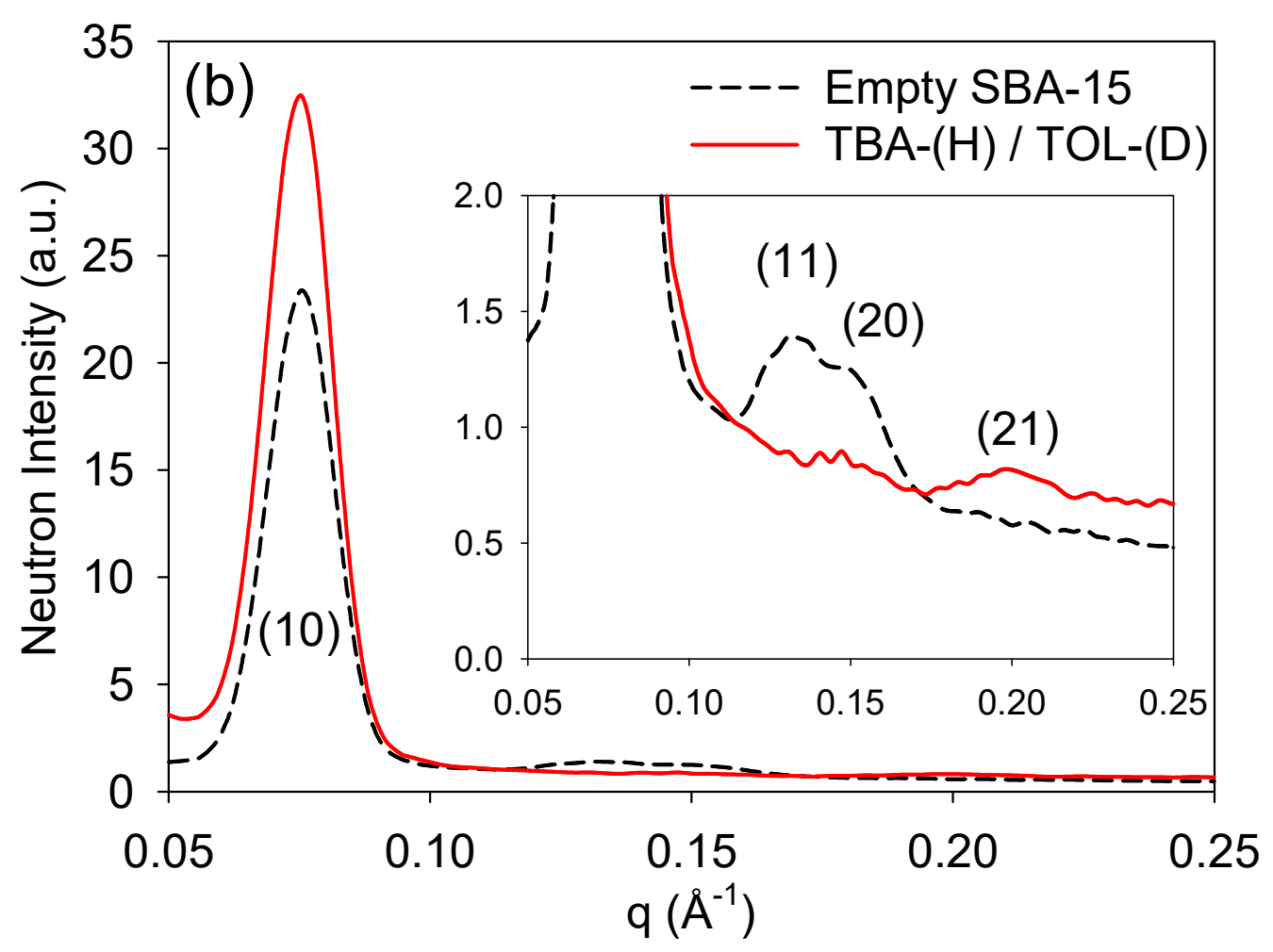




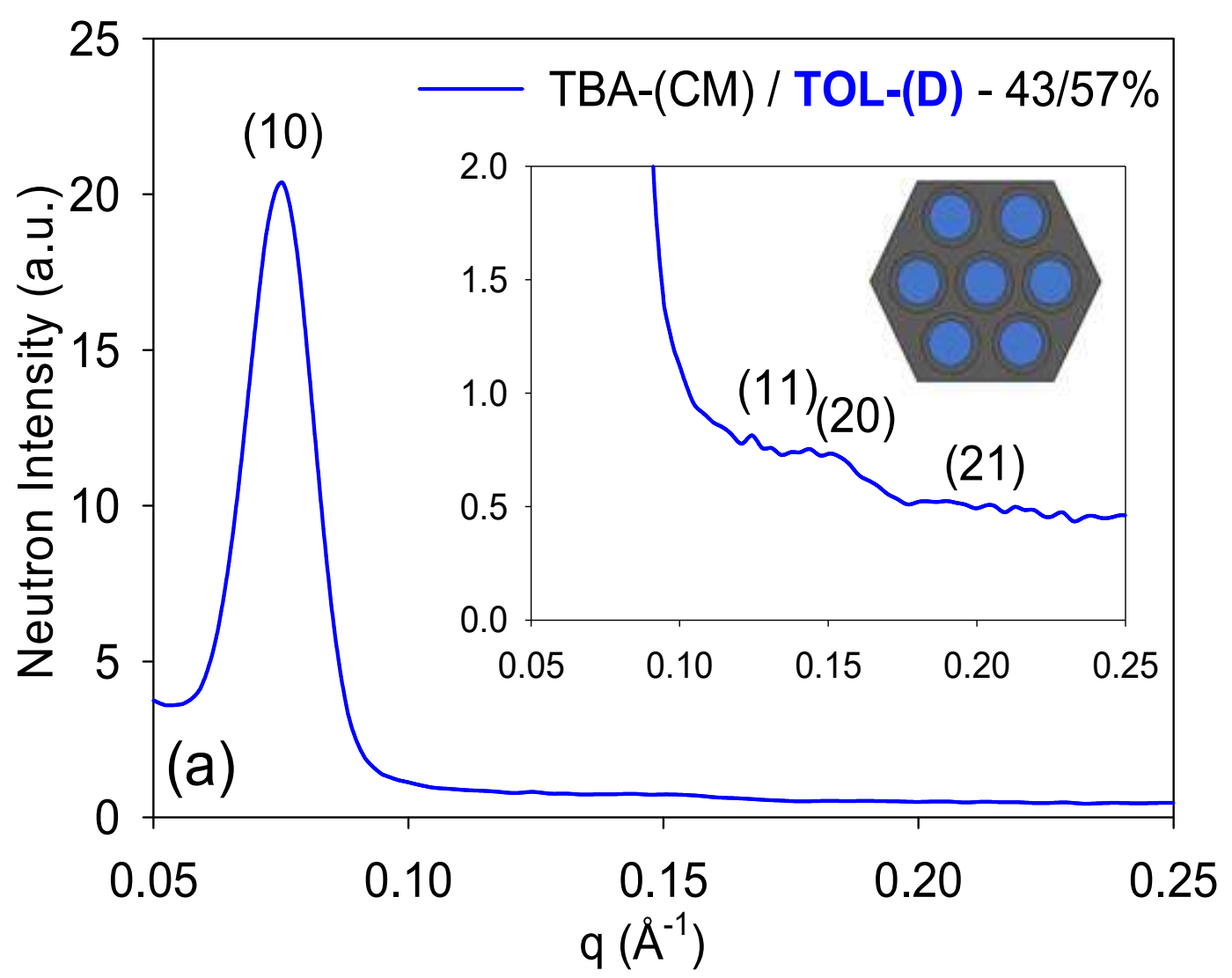




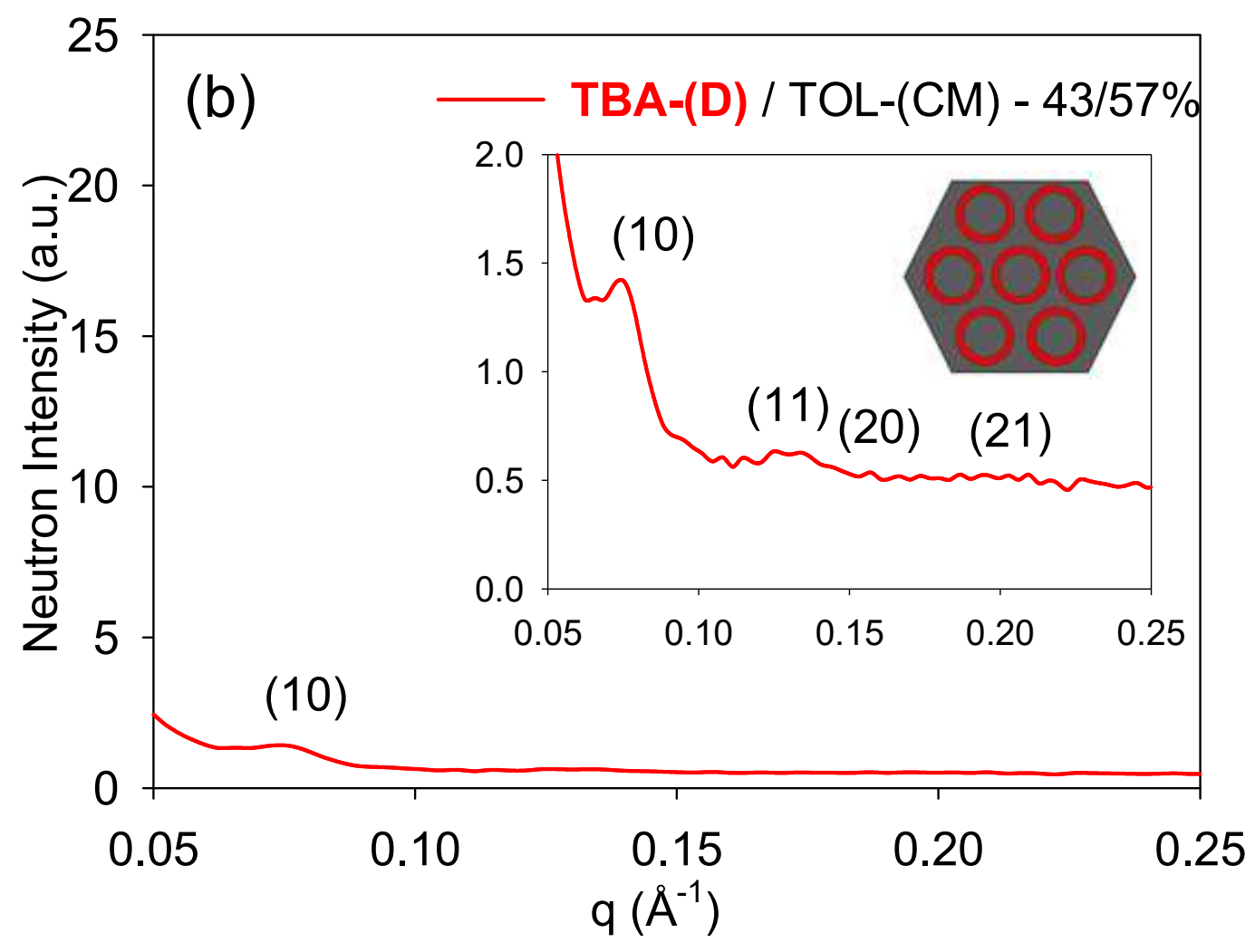




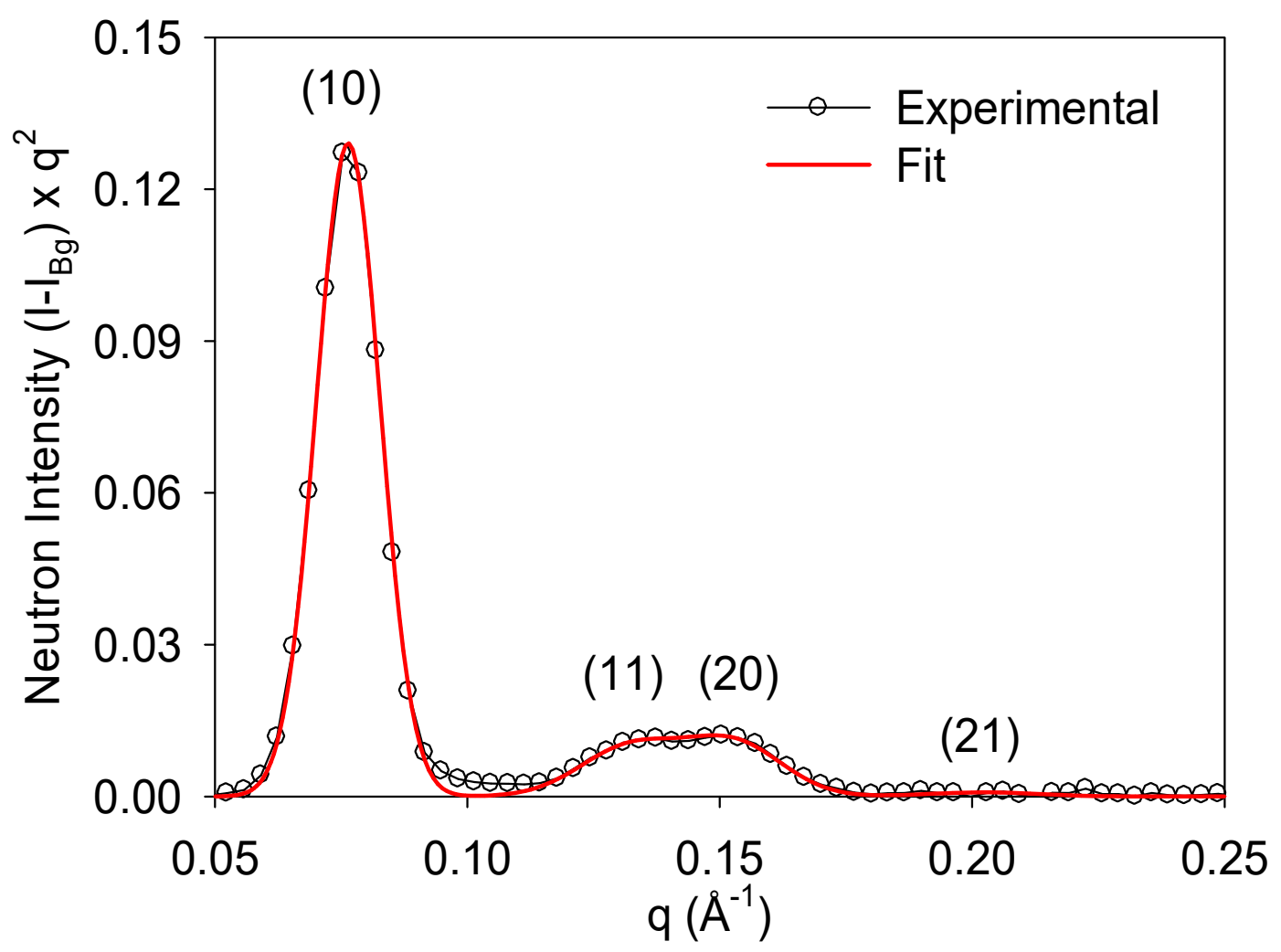




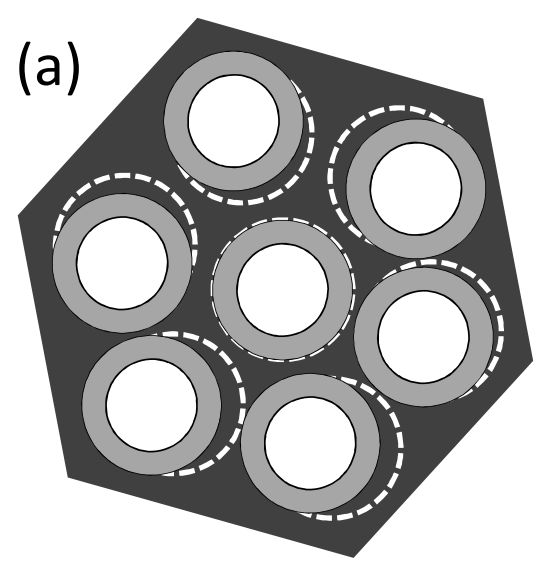


(b)

Silica

Shell

Core

$\rho_{\text {core }}$

$\rho_{\text {shell }}$ 


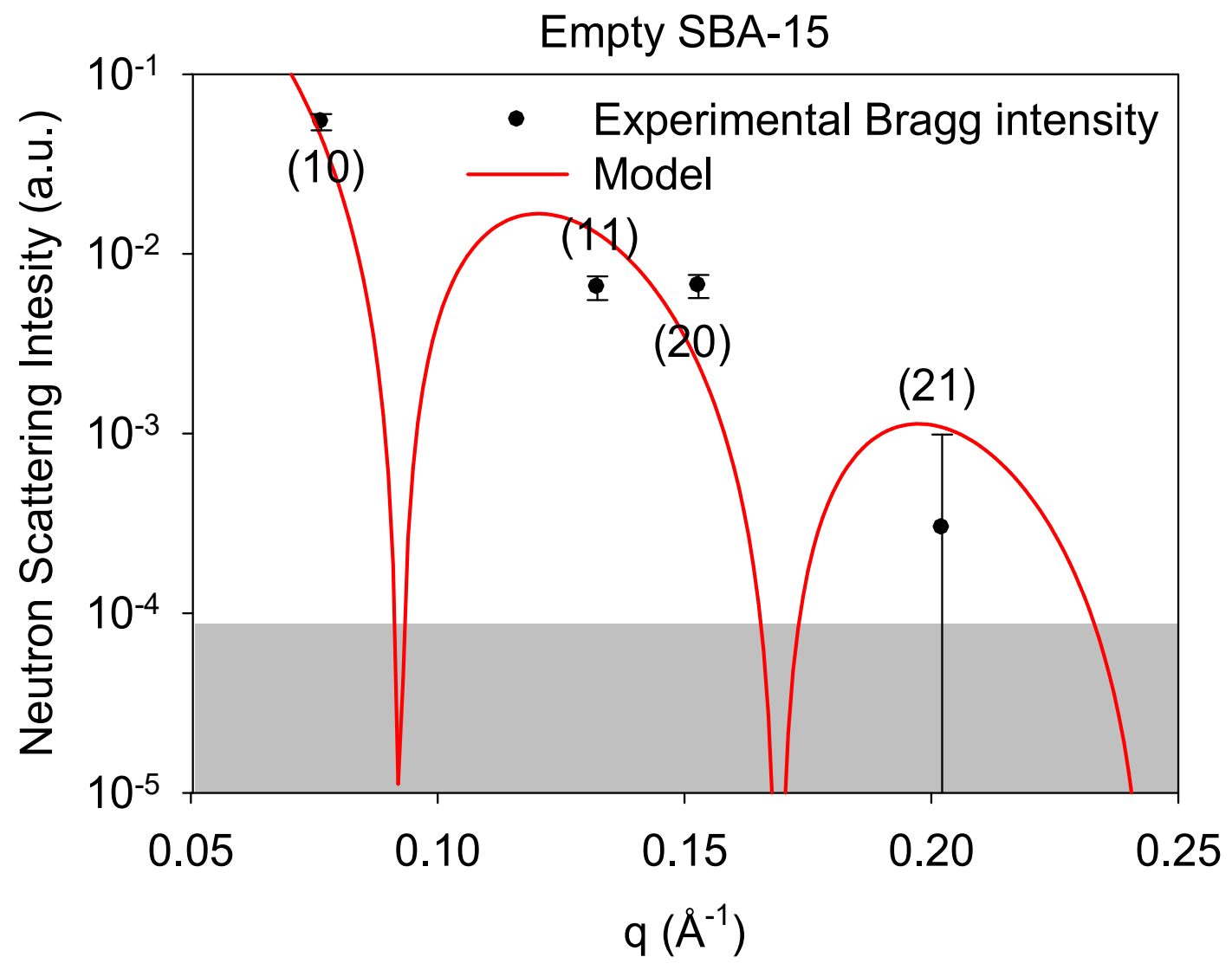




\section{Homogeneous}
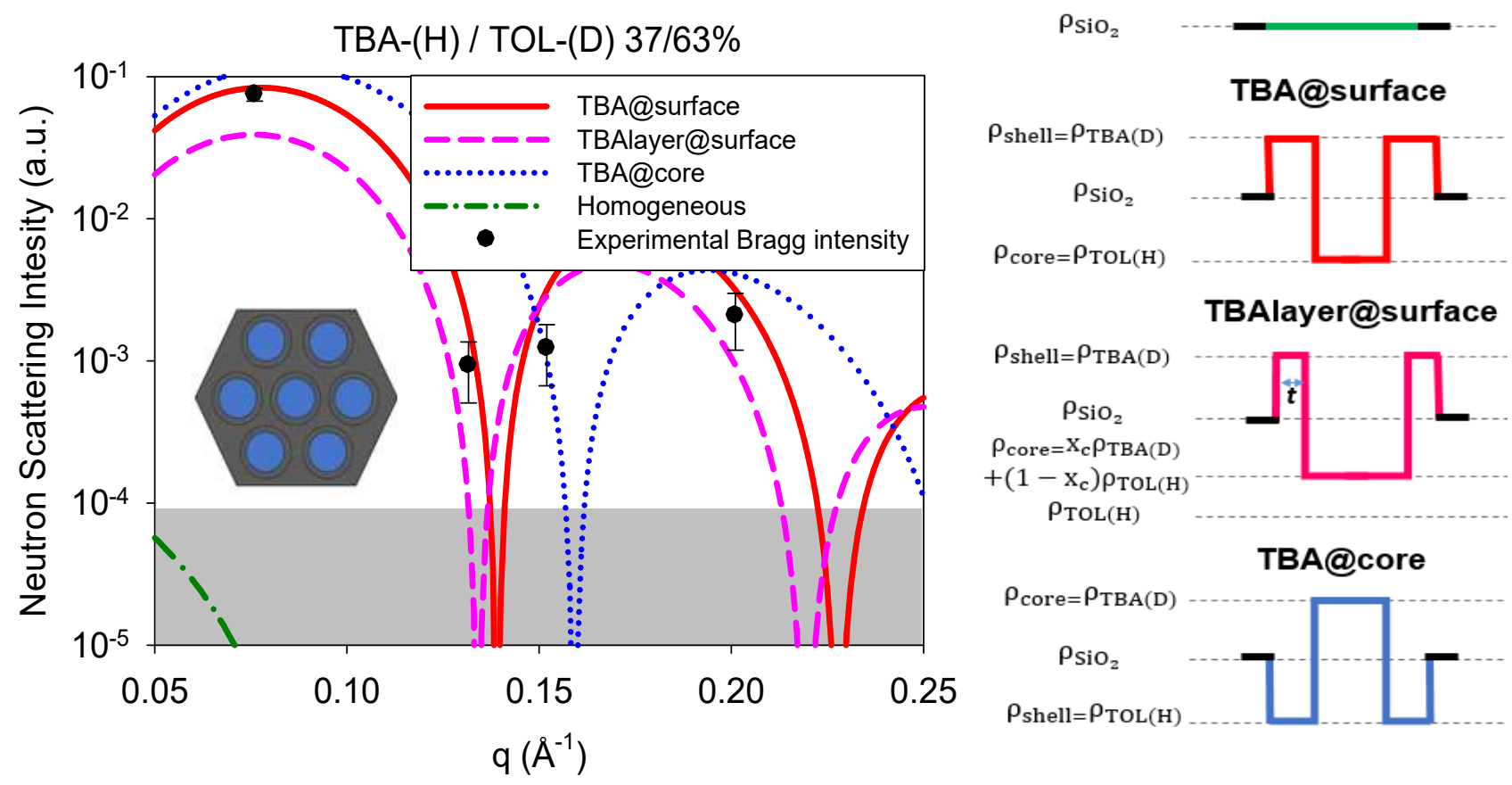

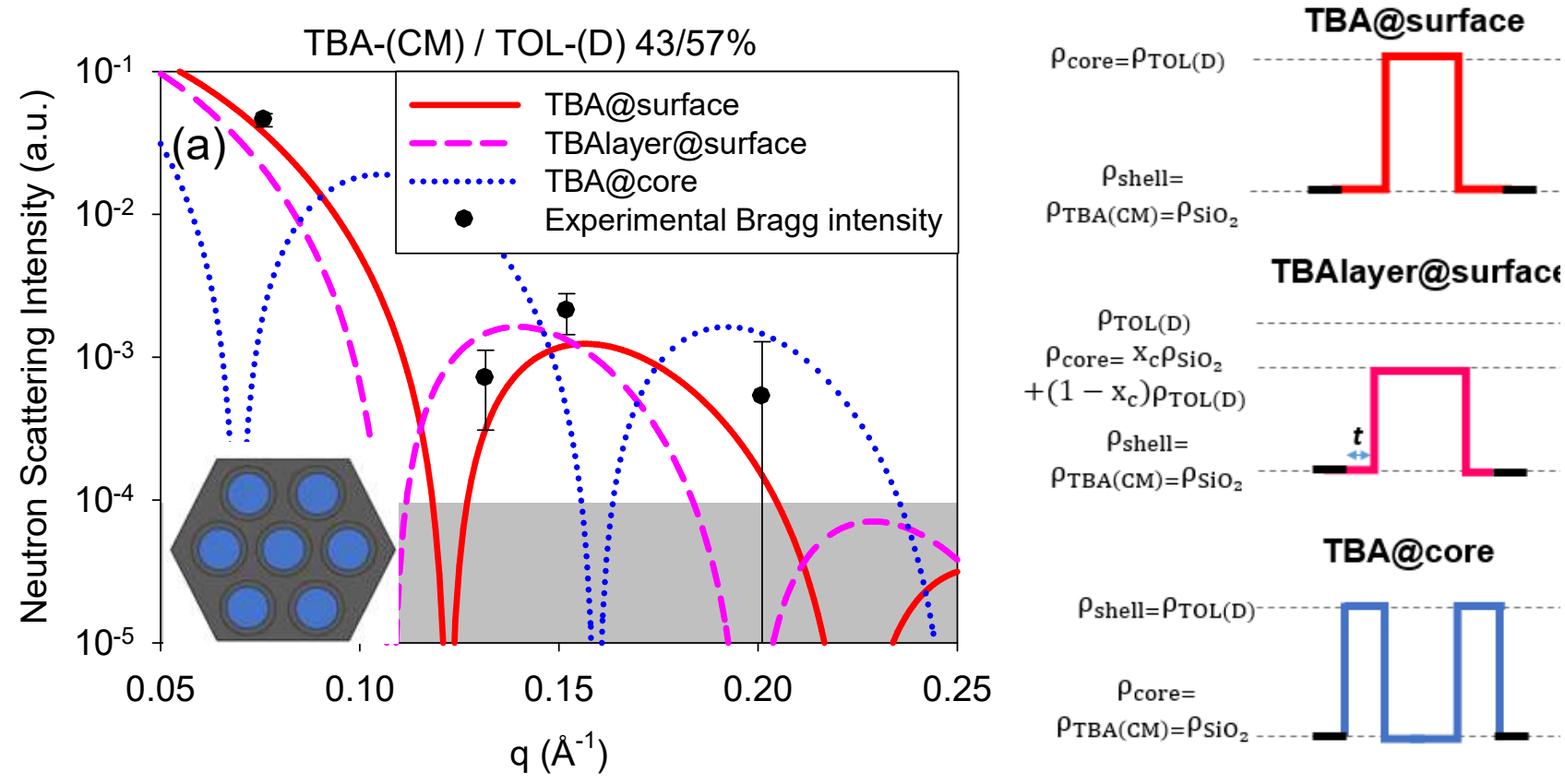

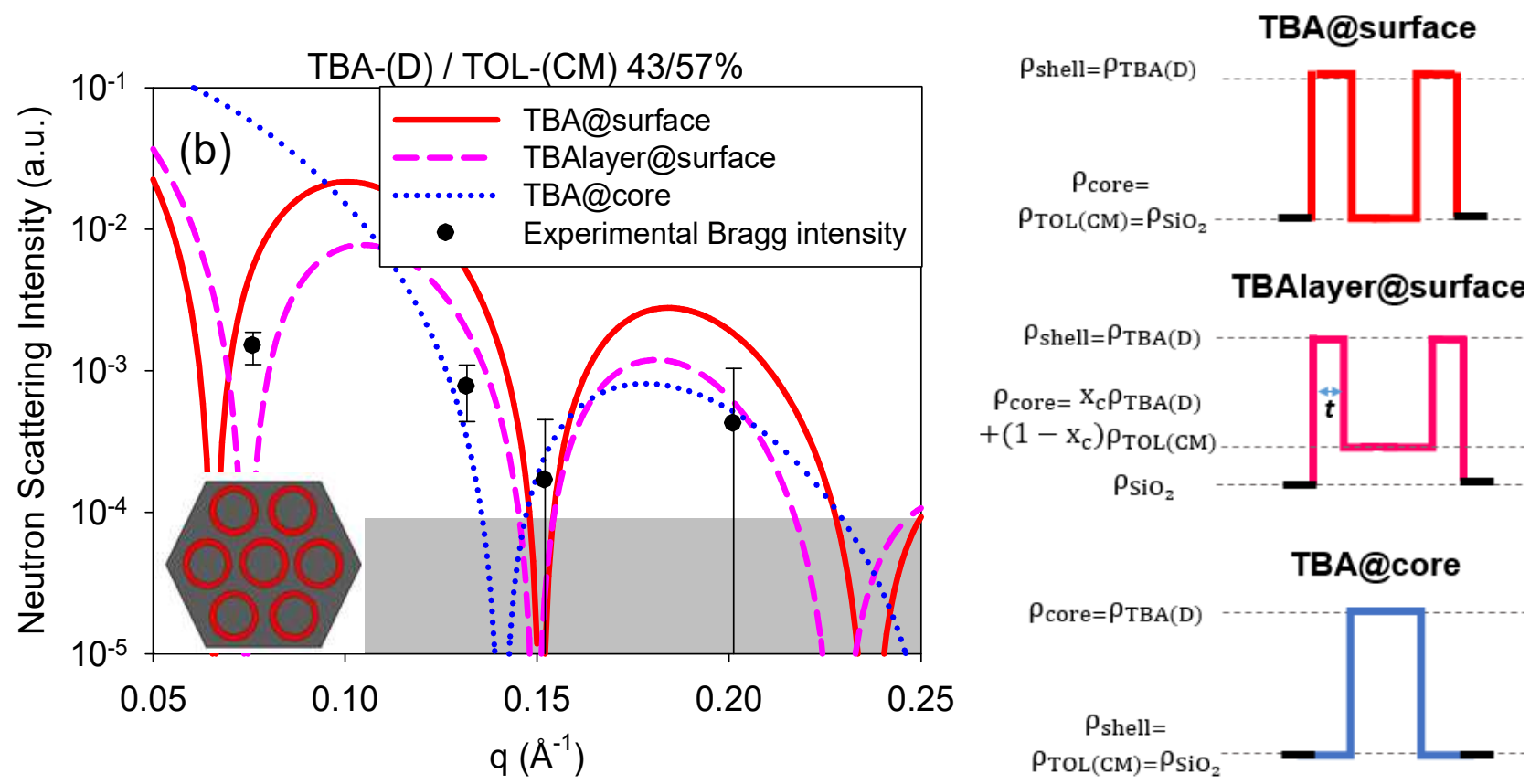


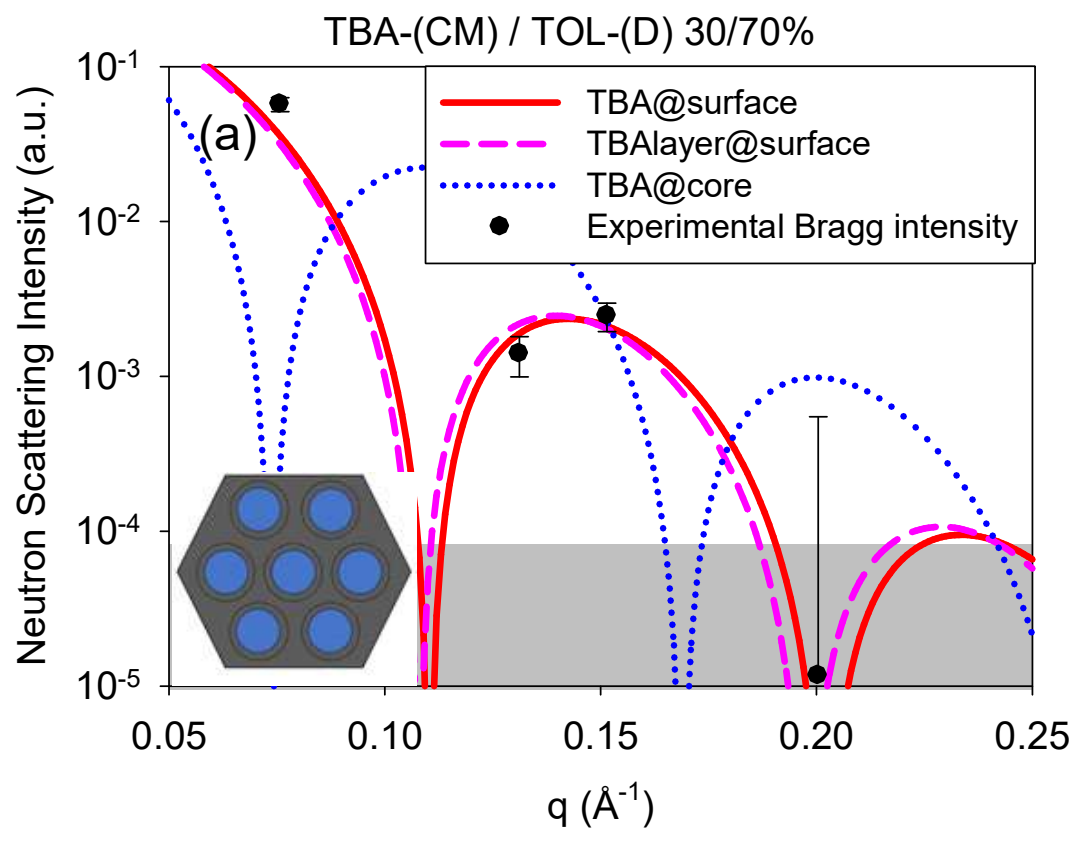

TBA@surface

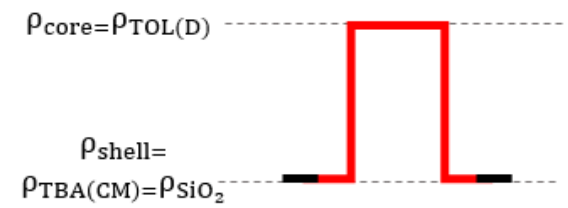

TBAlayer@surface

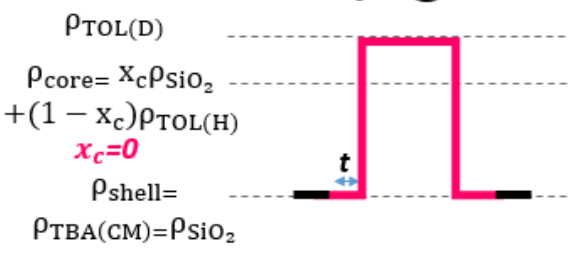

TBA@core

$\rho_{\text {shell }=} \rho_{\text {TOL(D) }} \cdots \cdots$
$\rho_{\text {core }=}$
$\rho_{\text {TBA(CM) }=} \rho_{\mathrm{SiO}_{2}} \cdots$ 

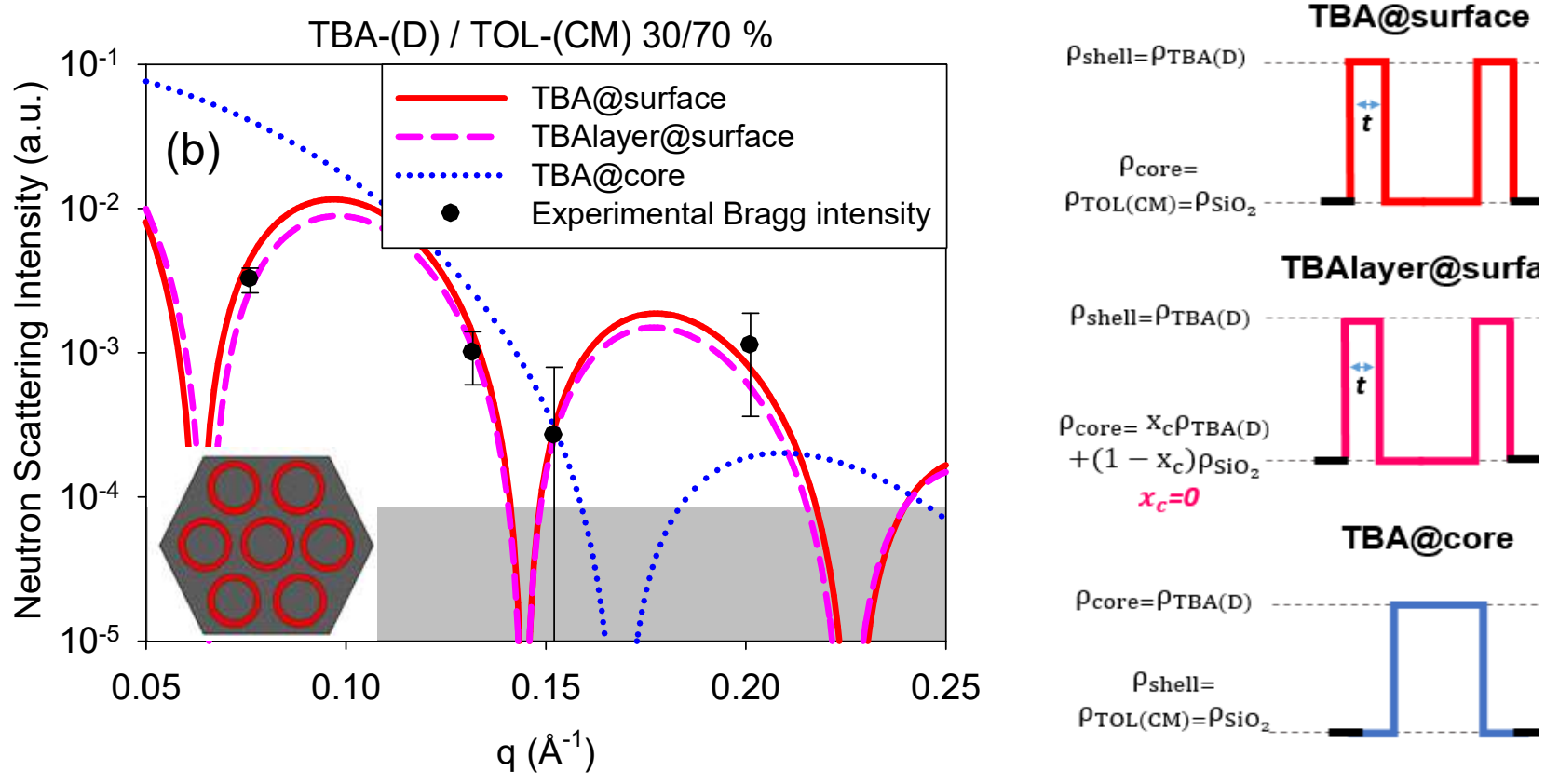